\title{
Modernising tactile acuity assessment; clinimetrics of semi- automated tests and effects of age, sex and anthropometry on performance
}

\author{
Nick A Olthof ${ }^{1,2}$, Michel W. Coppieters ${ }^{2,3}$, G Lorimer Moseley ${ }^{4}$, Michele Sterling ${ }^{5,6}$, Dylan J Chippindall ${ }^{2}$, Daniel S \\ Harvie ${ }^{\text {Corresp. 1,2 }}$ \\ ${ }^{1}$ School of Health Sciences and Social Work, Griffith University, Brisbane and Gold Coast, QLD, Australia \\ 2 Menzies Health Institute Queensland, Griffith University, Brisbane and Gold Coast, QLD, Australia \\ 3 Amsterdam Movement Sciences, Faculty of Behavioural and Movement Sciences, Vrije Universiteit Amsterdam, Amsterdam, The Netherlands \\ 4 IIMPACT in Health, University of South Australia, Adelaide, SA, Australia \\ 5 RECOVER Injury Research Centre, The University of Queensland, Brisbane, QLD, Australia \\ 6 NHMRC Centre of Research Excellence in Road Traffic Injury Recovery, The University of Queensland, Brisbane, QLD, Australia \\ Corresponding Author: Daniel S Harvie \\ Email address: d.harvie@griffith.edu.au
}

Background: Reduced tactile acuity has been observed in several chronic pain conditions and has been proposed as a clinical indicator of somatosensory impairments related to the condition. As some interventions targeting these impairments have resulted in pain reduction, assessing tactile acuity may have significant clinical potential. While two-point discrimination threshold (TPDT) is a popular method of assessing tactile acuity, large measurement error has been observed (impeding responsiveness) and its validity has been questioned. The recently developed semi-automated 'imprint Tactile Acuity Device' (iTAD) may improve tactile acuity assessment, but clinimetric properties of its scores (accuracy score, response time and rate correct score) need further examination.

Aims: Experiment 1: To determine inter-rater reliability and measurement error of TPDT and ITAD assessments. Experiment 2: To determine internal consistencies and floor or ceiling effects of ITAD scores, and investigate effects of age, sex, and anthropometry on performance.

Methods: Experiment 1: To assess inter-rater reliability $\left(\mathrm{ICC}_{(2,1)}\right)$ and measurement error (coefficient of variation (CoV)), three assessors each performed TPDT and ITAD assessments at the neck in forty healthy participants. Experiment 2: To assess internal consistency $\left(\operatorname{ICC}_{(2, k)}\right)$ and floor or ceiling effects (skewness z-scores), one hundred healthy participants performed the iTAD's localisation and orientation tests. Balanced for sex, participants were equally divided over five age brackets (18-30, 31-40, 41-50, 51-60 and 61-70). Age, sex, body mass index (BMI) and neck surface area were assessed to examine their direct (using multiple linear regression analysis) and indirect (using sequential mediation analysis) relationship with iTAD scores.

Results: Mean ICC $(2,1)$ was moderate for TPDT $(0.70)$ and moderate-to-good for the various iTAD scores (0.65-0.86). The CoV was $25.3 \%$ for TPDT and ranged from $6.1 \%$ to $16.5 \%$ for iTAD scores. Internal consistency was high for both iTAD accuracy scores $\left(\operatorname{ICC}_{(2,6)}=0.84 ; \operatorname{ICC}_{(2,4)}=0.86\right)$. No overt floor or ceiling effects were detected (all skewness z-scores < 3.29). Accuracy scores were only directly related to age (decreasing with increasing age) and sex (higher for men). 
TPDT indicating a potential for better responsiveness to treatment effects. Further, unlike previously reported for TPDT, ITAD scores appeared independent of anthropometry, which simplifies interpretation. Additionally, the iTAD assesses multiple aspects of tactile processing which may provide a more comprehensive evaluation of tactile acuity. Taken together, the iTAD shows promise in measuring tactile acuity, but patient studies are needed to verify clinical relevance. 
Modernising tactile acuity assessment; clinimetrics of semi-automated tests and effects of age, sex and anthropometry on performance.

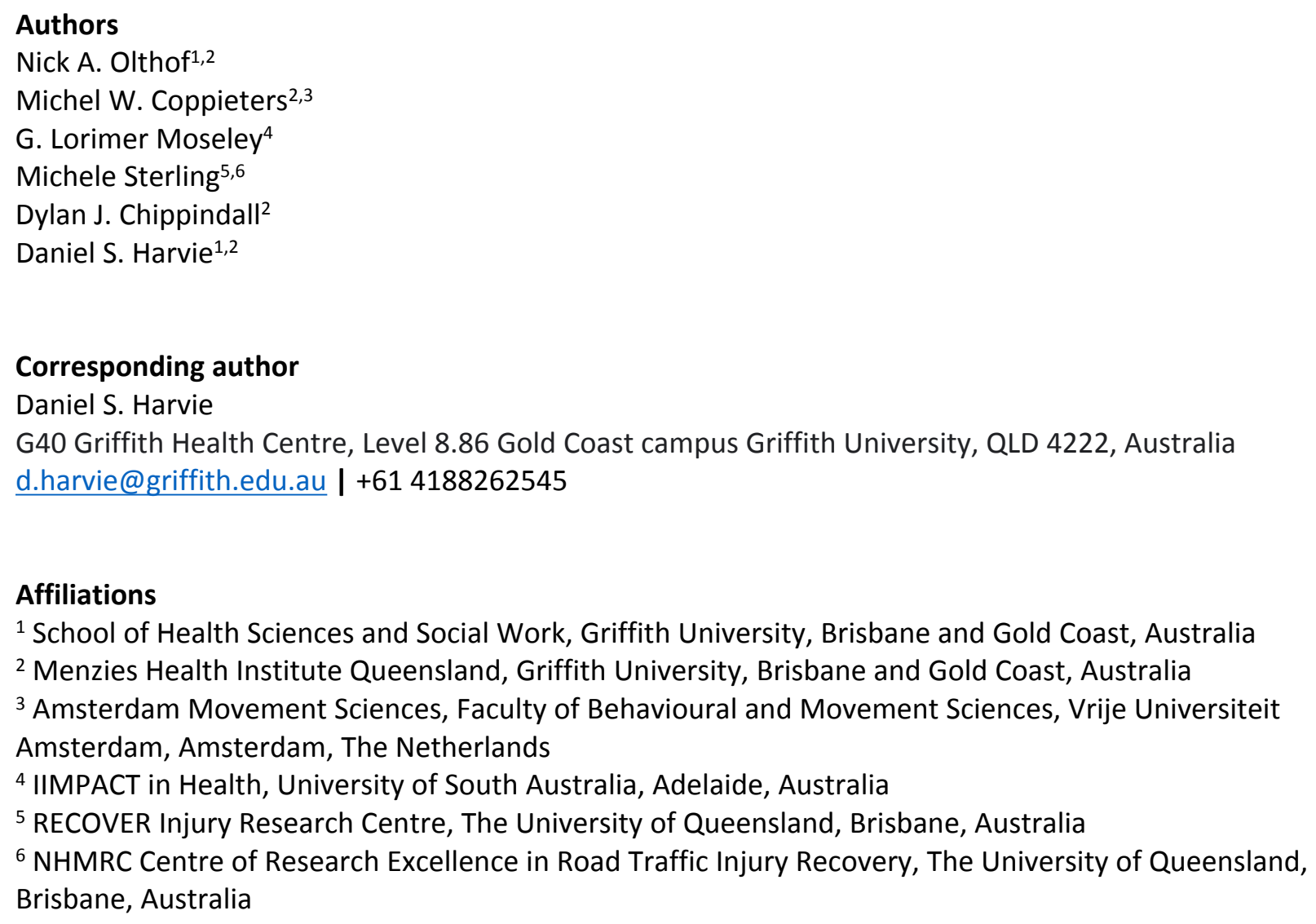

Affiliations

${ }^{1}$ School of Health Sciences and Social Work, Griffith University, Brisbane and Gold Coast, Australia

${ }^{2}$ Menzies Health Institute Queensland, Griffith University, Brisbane and Gold Coast, Australia

${ }^{3}$ Amsterdam Movement Sciences, Faculty of Behavioural and Movement Sciences, Vrije Universiteit Amsterdam, Amsterdam, The Netherlands

${ }^{4}$ IIMPACT in Health, University of South Australia, Adelaide, Australia

${ }^{5}$ RECOVER Injury Research Centre, The University of Queensland, Brisbane, Australia

${ }^{6}$ NHMRC Centre of Research Excellence in Road Traffic Injury Recovery, The University of Queensland, Brisbane, Australia

\section{ORCID}

Olthof: 0000-0002-6045-101X

Coppieters: 0000-0002-3958-4408

Moseley: 0000-0002-3750-4945

Sterling: 0000-0003-1772-2248

Harvie: 0000-0001-7693-4158

37 


\section{Background}

Measures of tactile acuity have been utilised to identify somatosensory impairments in a variety of painful conditions, such as musculoskeletal disorders [1-3], chronic pain [4, 5], amputation [6, 7], neuropathy [8], stroke [9], arthritis [10], complex regional pain syndrome [11] and spinal cord injury [12]. Patients with persistent pain typically demonstrate poorer tactile acuity than healthy controls [4], which is thought to reflect changes in somatosensory processing related to the condition. Since some interventions targeting these impairments have shown promise [13-17], assessing tactile acuity may have significant clinical potential.

Tactile acuity refers to the accuracy and clearness of touch perception [18]. Several dimensions of tactile acuity have been identified, such as two-point (or gap) discrimination, point (mis)localisation, length discrimination, orientation discrimination and shape/texture recognition [19-21]. As cutaneous mechanoreceptors respond to a variety of tactile stimuli [22], tactile acuity measures apply either dynamic deformation (movement), indentation (pressure) or vibration to the skin [23].

The two-point discrimination threshold (TPDT), i.e., the minimum distance between two tactile stimuli that can be perceived as spatially distinct, is the most frequently used and studied measure of tactile acuity $[4,8,24]$. Despite its popularity, its validity has been questioned due to involvement of non-spatial cues [25-28], and observations of abnormal scores [26, 27], hindering its interpretation. Moreover, TPDT scores appear affected by age [19, 21, 29, 30], sex [30, 31], and anthropometry (e.g., body mass index (BMI), waist-hip ratio, surface area of the tested body part) [31, 32], which further complicates interpretation of results. Although moderate to good intra-rater and inter-rater reliability has been reported, large measurement error has been observed [33], making it difficult to detect treatment effects. Correspondingly, TPDT was the least responsive sensibility test following median nerve injury and repair $[8,34]$, as well as the least responsive to improvements in hand function after surgery [2].

A variety of tests have recently been developed to improve tactile acuity assessment, such as the two-point orientation test [28], point-to-point test [35], tactile acuity charts [36], grating orientation task [37], and two-point estimation task [38, 39]. Additionally, technological developments instigated semi-automated tests which may be less affected by inter-rater variability $[7,9,40]$. An added benefit of these semi-automated tools is the potential for independent sensory training. This may be of clinical relevance, given that treatment success in manual sensory discrimination interventions could be limited by the need for caregiver involvement during at home training [41]. However, despite neck pain being 
70 ranked among the top five leading causes of years lived with disability globally [42], only a limited

71 number of these novel procedures have been applied to the neck [39, 43-45]. As such, the body of

72 knowledge about tactile acuity in neck pain appears limited compared to other painful conditions [46].

73 The development of improved tactile acuity assessment at the neck therefore has the potential to

74 elucidate mechanisms of neck pain, inform development of new treatment strategies, and guide clinical

75 decision making.

76 One recently developed tool designed to assess tactile acuity at the neck is the 'Imprint Tactile 77 Acuity Device' (ITAD) [47]. The ITAD is a semi-automated device that uses single and successive vibrotactile stimuli to quantify absolute and relative tactile localisation performance (for details see elsewhere [47]). As stimulus administration is automated and does not require synchronicity between locations, the iTAD may overcome some complications associated with TPDT. Despite some initial design issues, the iTAD prototype has shown comparable intra-rater reliability to existing tests [47], suggesting prospective utility. In this manuscript, we report two experiments that investigate the clinimetric properties of an updated version of the ITAD. Experiment 1 aimed to determine inter-rater reliability and measurement error of both the ITAD and TPDT assessments, which is currently the most reliable tactile acuity assessment at the neck [43]. Experiment 2 aimed to quantify internal consistency and identify floor or ceiling effects of the iTAD scores, and determine their relationship with age, sex, BMI and neck surface area.

\section{Methods}

Both experiments were approved by Griffith University Human Research Ethics committee (\#2016/168), and all participants gave written informed consent prior to participating.

2.1 Experiment 1: Inter-rater reliability and measurement error

\subsubsection{Design and procedure}

A test-retest study investigated the inter-rater reliability and measurement error of the ITAD and TPDT. Three assessors each performed both assessments in a private, quiet, room. The order of assessments was randomised between participants, but consistent between assessors for each 
102 minute break between assessors and were blinded to their scores. Assessors were blinded to all iTAD

103 scores and TPDT scores of the other assessors. The anatomical location of both assessments was

104 standardised but independently determined by each assessor.

105

\subsubsection{Participants}

Using a convenience sample, individuals without current pain and neurological symptoms as well as without a history of persistent (i.e., $>3$ months) pain and neurological symptoms in the past five years were recruited from the general public.

\subsubsection{Assessors}

112 Three final year physiotherapy Masters students each performed iTAD and TPDT assessments after 113 receiving approximately one hour of training for each procedure. Test instructions and performance

114 were standardised using a testing protocol. Training included approximately 20-30 minutes of

115 instructions on the testing protocol and about 30-40 minutes of practicing the protocol. Each assessor

116 practised each protocol five times and underwent each assessment at least once.

118 2.1.4 TPDT assessment

119 A digital caliper (Renegade industrial, carbon fibre, RCFVC150) was used to establish TPDT, utilizing 120 the two arms and pressure of its own weight as tactile stimuli. Contact area of the tip of each arm was 121 approximately $0.25 \mathrm{~mm} \times 0.5 \mathrm{~mm}$ and stimulus duration $<1$ second. During TPDT assessment, participants 122 were seated with their forehead resting on a table in front of them. On the dominant side, TPDT was 123 measured in a cranio-caudal direction with the caudal arm of the caliper stationary at $15 \mathrm{~mm}$ lateral to 124 the spinous process of $\mathrm{C7}$.

125 Similar to a previously published procedure [48], a two-alternative forced-choice (one or two 126 points) staircase method was used, alternating two ascending and two descending runs (see Fig. 1). The 127 caliper distance started at $15 \mathrm{~mm}$, increasing with $5 \mathrm{~mm}$ steps. Steps were reduced to $2 \mathrm{~mm}$ for the 128 subsequent runs. To avoid guessing, three consecutive reports of either one or two points indicated a 129 reversal. A $10 \mathrm{~mm}$ step was added in the direction of the completed run, before running the reversed 130 direction. The TPDT was calculated by averaging the scores of the four reversals, expressed in 131 millimetres, with larger distances indicating poorer tactile acuity. 


\subsection{5 iTAD assessment}

136 iTAD prototype

137 The iTAD consists of a wearable neoprene collar containing twelve vibrotactile stimulators $(\sim 200 \mathrm{~Hz}$

138 with $0.75 \mathrm{~g}$ vibration amplitude), arranged in three rows of four (see Fig. 2). A wirelessly connected

139 tablet operates the stimulators and records user's responses. After fitting and familiarisation, two tactile

140 acuity tests were performed: the localisation test, which measures the ability to localise the vibrations

141 (one second stimulus duration), and the orientation test, which measures the ability to determine the

142 orientation of two successive adjacent vibrations relative to each other ( 0.7 seconds stimulus duration

143 each). Accuracy scores (i.e., percentage correct) for each test, and the overall score (i.e., mean of both

144 test), were calculated with higher scores indicating better tactile acuity. For a full description of the iTAD 145 prototype and its assessment procedures, see elsewhere [47].

Changes to the prototype

150 After development of the prototype, the internode distance has been reduced to $32.5 \mathrm{~mm}$ (centre-

151 to-centre) between all rows and columns. Additionally, a layer of foam was placed in the collar to aid

152 fitting consistency. For the localisation test, the number of trials was increased from 48 to 72 , delivered 153 in six series of twelve. For the orientation test, the number of trials was increased from 48 to 64 ,

154 delivered in four series of sixteen. Furthermore, trials were block randomised within each series,

155 alternating between sides of the neck.

\section{Additional scores}

158 In order to better quantify tactile acuity, two new scores were added. For each test, and the overall score, (average) response time was recorded in milliseconds with lower scores indicating faster responses. Additionally, a rate correct score was calculated ( $=\sum$ (correct responses) $/ \Sigma$ (response times in minutes)), quantifying the number of correct responses per minute of response activity [49] with higher scores indicating better tactile acuity. As the rate correct score integrates response time and accuracy, it accounts for individual speed-accuracy trade-off strategies and has been suggested to provide a better estimate of perceptual performance [49]. 
166

167

168

169

170

171

172

173

174

175

176

177

178

179

180

181

182

183

184

185

186

187

188

189

190

191

192

193

194

195

196

\subsubsection{Statistical analyses}

Inter-rater reliability was assessed by calculating the intraclass correlation coefficient, model 2,1 $\left(\operatorname{ICC}_{(2,1)}\right)$ (i.e., two-way random, absolute agreement, single measures). Values were interpreted as poor $(<0.5)$, moderate (0.5-0.74), good (0.75-0.89) or excellent $(\geq 0.9)[50]$.

The standard error of measurement (SEM) was calculated using variance components from the ANOVA table $\left(\mathrm{V}\left(\sigma^{2}{ }_{\text {observer }}+\sigma^{2}\right.\right.$ residual $\left.)\right)$ to assess measurement error [51]. As TPDT and ITAD use different metric units, the SEM was additionally converted to the coefficient of variation (COV) (i.e., SEM as a percentage of mean score) allowing direct comparison of measurement error [52]. Since both $<10 \%$ and $<20 \%$ have been suggested as a good CoV $[53,54]$, results were categorised into: $<10 \%, 10-20 \%$ and $>20 \%$.

To assist clinical interpretation of the SEM, the smallest detectable change with a $95 \%$ confidence interval (SDC_95) was calculated (1.96*V2*SEM) [51]. Additionally, SDC values with smaller confidence intervals (SDC_80, SDC_85 and SDC_90) were computed (replacing 1.96 with respective z-values).

\subsubsection{Sample size calculation}

To detect a hypothesized ICC $(2,1)$ of 0.7 with three repeated measurements (recommended to optimise sample size [55]), while $\alpha=0.05, \beta=0.2$ and $\mathrm{ICC}_{(2,1)}=0.5$ for the null hypothesis, a minimum of 40 participants is required [55]. Therefore, we aimed to recruit 40 participants.

2.2 Experiment 2: Internal consistency, floor and ceiling effects, and relationship with age, sex and anthropometry

\subsubsection{Design and procedure}

Using a cross-sectional design, the internal consistencies of the localisation and orientation accuracy score were investigated. Additionally, floor and ceiling effects of all iTAD scores were assessed, as well as their relationship with age, sex, BMI and neck surface area. In one session, participants performed both iTAD tests after age, sex, and anthropometric measures (see 2.2.4) were recorded. Measures were taken by a single assessor in a private, quiet room. The assessor had several hours of prior experience performing iTAD assessments.

\subsubsection{Participants}

Peer) reviewing PDF | (2021:05:60922:1:0:NEW 28 Jul 2021) 
197 Recruitment and selection criteria were identical to experiment one. However, participants

198 represent a different cohort without overlap.

199

200

2.2.3 iTAD tests

201

For procedure of the iTAD tests, see 2.1.5.

202

203

\subsubsection{Anthropometric measurements}

After weight $(\mathrm{kg})$ and height $(\mathrm{m})$ were recorded, BMI was calculated (weight/height ${ }^{2}$ ). Using a tape measure, the distance from the caudal aspect of the external occipital protuberance to the spinous process of $\mathrm{C7}$ was measured to quantify neck length $(\mathrm{cm})$. Neck circumference $(\mathrm{cm})$ was measured at half-way of the neck length measurement placing the tape measure horizontally around the neck. Using these measurements, the posterior neck surface area was estimated (neck length*(neck circumference/2)).

210

\subsubsection{Statistical analysis}

Internal consistency was assessed by calculating the inter-relatedness of accuracy scores between series within each test, using ICC model 2,k (i.e., two-way random, absolute agreement, average measures). The $\operatorname{ICC}_{(2, \mathrm{k})}$ was chosen over the Cronbach's alpha, the equivalent of the $\operatorname{ICC}_{(3, \mathrm{k})}$ (i.e., two-way random, consistency, average measures), to include absolute differences between series. Although various cut-offs are proposed, most recommend 0.7-0.9 for high internal consistency [56].

For the accuracy scores, floor and ceiling effects were considered present if $>15 \%$ of the participant scored within either the highest or lowest $20 \%$ of the scale [57]. However, such assessment would not be adequate for response times or rate correct scores, as their scales have no limit on one end and scores are (near) impossible at the other. Therefore, floor and ceiling effects for all iTAD scores were assessed by calculating z-scores for the skewness of their distribution (i.e., the skew value divided by its standard error) $[58,59]$. Floor and ceiling effects may be present with a $z$-score $> \pm 1.96$ in small $(n<50)$ or $> \pm 3.29$ in medium $(50<n<300)$ sized samples $[58,59]$.

In order to study the direct multivariate relationships of age, sex, BMI and neck surface area with the localisation and orientation accuracy score, multiple linear regressions (enter models) were performed. Furthermore, to estimate the potential indirect effects of age and sex through BMI and/or neck surface area, sequential mediation analyses were performed using the SPSS extension PROCESS (model 6; 5000 bootstrapped samples) as a secondary analysis. Mediation analyses for all other iTAD 
229

230

231

232

233

234

235

236

237

238

239

240

241

242

243

244

245

246

247

248

249

250

251

252

253

254

255

256

257

258

259

260

scores were performed as exploratory analyses. Regression models are expressed in (adjusted) explained variance $\left(\mathrm{R}^{2}\right.$ adjusted $)$. For all relationships, both the mean unstandardized regression coefficient (b) and the semi-partial correlation (sr) are provided. Indirect effects are expressed in percentage mediation $\left(P_{m}\right)$.

\subsubsection{Sample size}

To examine internal consistency, a minimum of 100 participants is recommended [57]. To explore potential floor and ceiling effects, a minimum of 50 participants is recommended [57]. For the multiple linear regressions, 100 participants were needed to find a medium sized $\left(f^{2}=0.15\right)$ prediction model using four predictors with a Bonferroni corrected p-value of 0.025 and $80 \%$ power. Taken together, the sample size was set for 100 participants, with 10 participants of both sexes in each of five age brackets (18-30, 31-40, 41-50, 51-60 and 61-70).

\section{Results}

\subsection{Experiment 1: Inter-rater reliability and measurement error}

Forty individuals (25 male) participated, with a mean (SD) age of 24.1 (4.5) years. One participant was left hand dominant and the others right hand dominant. Mean scores, inter-rater reliabilities and measurement errors are displayed in Table 1. Inter-rater reliability was good for ITAD orientation accuracy score, overall accuracy score, and all response times. All other scores displayed moderate interrater reliability. The CoV was $<10 \%$ for all iTAD response times and $>20 \%$ for TPDT. All other scores had a CoV of $10-20 \%$.

\section{----- INSERT TABLE 1 ABOUT HERE ------}

For all scores, the SDC is presented with varying confidence intervals (80\% to $95 \%$ ) in Table 2. Each can be used to assess the chance that an observed change in score, when larger in either direction, could reflect measurement error: (100\% - confidence interval)/2 (i.e., $<10 \%,<7.5 \%,<5 \%$ and $<2.5 \%$ respectively). For example, a change of $+16.2 \%$ in localisation accuracy score would have a $7.5-10 \%$ chance to be a result of measurement error, whereas a change of $+21.3 \%$ a $2.5-5 \%$ chance. 
261

262

263

264

265

266

267

268

269

270

271

272

273

274

275

276

277

278

279

280

281

282

283

284

285

286

287

288

289

290

291

292

----- INSERT TABLE 2 ABOUT HERE ------

3.2 Experiment 2: Internal consistency, floor and ceiling effects, and relationship with age, sex and anthropometry

One hundred individuals participated, with ten of both sexes per predetermined age bracket. Ten participants were left hand dominant, 88 right hand dominant and two were ambidextrous. Mean (SD) BMI was 26.4 (4.6) and mean (SD) neck surface area was $261.0 \mathrm{~cm}^{2}$ (48.1). Mean (SD) duration was 03:06 $(00: 25)$ minutes for the localisation test and 03:38 (00:28) for the orientation test.

\subsubsection{Internal consistency}

The $\operatorname{ICC}_{(2,6)}(95 \% \mathrm{Cl})$ for the localisation accuracy score was $0.84(0.79-0.89)$ and the $\operatorname{ICC}_{(2,4)}(95 \% \mathrm{Cl})$ for the orientation accuracy score was $0.86(0.81-0.90)$.

\subsubsection{Floor and ceiling effects}

None of the accuracy scores had $>5 \%$ of participants scoring in either the highest or lowest $20 \%$. Only localisation response time had a skewness $z$-score $> \pm 1.96(z=+2.73)$, which was still $< \pm 3.29$.

\subsubsection{Relationship with age, sex and anthropometry}

Multiple linear regression analyses showed significant prediction models for both the localisation accuracy score $\left(F(4,95)=4.92, p<0.01, R_{\text {adjusted }}=0.14\right)$ and orientation accuracy score $(F(4,95)=5.82$, $\left.\mathrm{p}<0.01, \mathrm{R}_{\text {adjusted }}^{2}=0.16\right)$.

For the localisation accuracy score, both age $(b=-0.20, s r=-0.20, p=0.03)$ and $\operatorname{sex}(b=8.04, s r=0.25$, $p=0.01)$ had a significant contribution to the model, whereas BMI $(p=0.11)$ and neck surface area $(p=0.89)$ did not. This indicates that, on average, men scored $8.04 \%$ higher than women, and that scores decreased by $0.20 \%$ for each year of age.

For the orientation accuracy score, both age $(b=-0.39, s r=-0.36, p=0.00)$ and $\operatorname{sex}(b=6.69, s r=0.19$, $p=0.04)$ contributed significantly to the model, whereas BMI $(p=0.71)$ and neck surface area $(p=0.94)$ did not. This indicates that, on average, men scored $6.69 \%$ higher than women, and that scores decreased by $0.39 \%$ for each year of age.

The mediation analyses indicated several significant relationships between demographic and anthropometric variables (see Fig. 3). However, for the localisation accuracy score, the total indirect effects of age $\left(P_{m}=0.16, p>0.05\right)$, and sex $\left(P_{m}=0.06, p>0.05\right)$, through $B M I$ and neck surface area were 
293

294

295

296

297 298

299

300

301

302

303

304

305

306

307

308

309

310

311

312

313

314

315

316

317

318

319

320

321

322

323

324

non-significant. Similarly, the total indirect effects of age $\left(P_{m}=0.02, p>0.05\right)$, and sex $\left(P_{m}=0.04, p>0.05\right)$ were also non-significant for the orientation accuracy score. Additionally, all individual indirect effects were non-significant for both tests. This indicates that BMI and/or neck surface area did not significantly mediate the effects of age or sex for either accuracy score. Scatterplots of localisation and orientation accuracy scores as a function of age and sex are displayed in Figure 4.

----- INSERT FIGURE 3 and 4 ABOUT HERE ------

Exploratory mediation analysis of the other ITAD scores showed similar patterns, other than sex not significantly predicting the localisation or orientation response time and rate correct score. Additionally, rate correct scores were more strongly predicted by age. Figures of all mediation analyses can be found in Supplemental files (Fig. S1-S3). Scatterplots of all iTAD scores as a function of age and sex can be also be found in Supplement files (Fig. S4, S5)

\section{Discussion}

Inter-rater reliability

Inter-rater reliability was moderate for TPDT and moderate to good for the ITAD scores. When directly compared, $I C C_{(2.1)}$ values were somewhat higher for iTAD's response times, but similar between

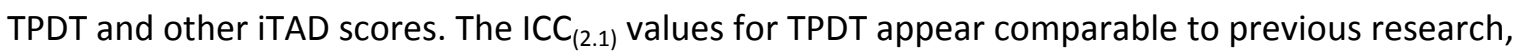
although results vary $[33,43,48,60,61]$. Compared to the prototype, reliability appears improved with the new generation ITAD despite previous values representing intra-rater reliability [47].

\section{Measurement error}

Results indicate a larger CoV for TPDT than all iTAD scores. For TPDT assessment, differences in speed, timing and intensity of stimulus delivery affect results [25, 27, 62]. Therefore, variability in these parameters between raters, trials, and both arms of the caliper, increases measurement error. An inherent problem of manual TPDT assessment is the inability to control, or assess, these variables in a clinical setting. The ITAD scores may be less prone to these sources of error variance, as stimulus administration is automated and does not require synchronicity between locations. Clinically, this implies less difficulty detecting change with iTAD assessments, potentially resulting in better 
325 responsiveness to treatment effects [57]. Notably, CoV for TPDT seems somewhat larger than previously 326 reported (17.4-20.6\% [48]; 19.1\% [33]). This may be due to the testing procedure (e.g. orientation of 327 caliper, number of reversals), for which no standard is available [60,61]. Yet, mean (SD) TPDT scores 328 appeared similar to several other reports (mean (SD) range: $45.9(18.4)$ to $62.6(22.9)$ ) [33, 39, 45, 63], 329 although somewhat higher than others (mean (SD) range: 21.7 (6.2) to $35.2(9.6)$ ) [43, 48, 64]. Further, 330 measurement error may depend on various factors related to both participants and assessors included

331 [33]. However, these were constant between the two assessments in this experiment, allowing for a 332 more direct comparison.

333 Several SDC values were also presented. Although conventional, the SDC_95 may provide high 334 specificity (few false positives) but low sensitivity (many false negatives) in detecting change due to its 335 large confidence interval [50]. As both false conclusions can negatively impact clinical decision making, 336 presenting a range of SDC values may support more precise interpretation of observed changes in 337 relation to measurement error.

338

Internal consistency

Despite including absolute differences between series, internal consistency was high for both the localisation and orientation accuracy scores and higher than for the ITAD prototype [47]. Internal consistency is frequently applied to questionnaires but underutilised in experimental tasks, mostly because scores cannot be split into multiple representative parts $[65,66]$. However, internal consistency has previously been established in measures such as electrocardiography [67], electroencephalography

345 [68], joint position sense [69] and motion analysis [70]. One benefit of reporting internal consistency as

346 a measure of reliability is its comparability between studies, even if only single measurements are taken 347 [65].

Floor and ceiling effects

350 No floor or ceiling effects, which may limit responsiveness [57], were detected in iTAD scores; only 351 a potential, yet debatable, floor effect for localisation response time. This could indicate difficulty in 352 detecting improvements in already fast responders. However, this may not be clinically relevant, as slow 353 responders are more likely targets for treatment.

354

355 Relationship with age, sex, BMI and neck surface area 
356

357

358

359

360

361

362

363

364

365

366

367

368

369

370

371

372

373

374

375

376

377

378

379

380

381

382

383

384

385

386

Results indicated that localisation and orientation accuracy scores were only directly related to age (decreasing with increasing age) and sex (lower for women). This implies that age and sex, but not BMI or neck surface area, should be considered when interpreting scores.

Regarding the effect of age, similar sized negative correlations between age and tactile acuity have previously been established using TPDT $[30,71]$. One frequently proposed mechanism is the decreased cortical inhibition in response to tactile stimulation associated with older age [29, 72-74]. Interestingly, these age-related declines in tactile acuity can potentially be reversed with sensory training $[74,75]$.

Concerning the effect of sex, previous reports seem inconsistent and vary between body regions when measured with TPDT [76]. For example, better tactile acuity has been reported for women at the orofacial region [77] and knee [31], for men at the knee [10], and no differences were found at the lower back $[10,71]$. To the best of our knowledge, this is the first study examining sex differences at the neck, making it difficult to compare results. Additionally, sex differences could dependent on task type. In a single experiment, women made more errors in a tactile object recognition task despite demonstrating similar TPDT scores [30].

For TPDT assessment, scores seem affected by BMI [31, 71] and body fat ratios [78]. One proposed mechanism is the sensitivity of TPDT scores to skin deformation $[62,78]$, which is affected by BMI [79]. The ITAD uses vibrotactile stimuli rather than indentation, which may explain the contrasting results.

Results also contrast previous reports indicating that tactile acuity at the fingertips relates to surface area, potentially due its relationship with mechanoreceptor density [32]. One explanation is that the utilized neck surface area assessment may be a poor proxy for receptive field configuration. Different to the fingertips, necks exhibit variation in hairy (vs. non-hairy) skin which typically does not contain Pacinian mechanoreceptors, known to be activated by high frequency vibrations [22]. Proportion of hairy skin may therefore moderate the relationship between neck surface area and iTAD scores, which was not investigated in this study. Alternatively, lack of a significant relationship with surface area could also indicate that ITAD scores may be less affected by peripheral receptive field configuration. Correspondingly, the ability to accurately localise tactile stimuli may be more centrally organised [80], and higher order cognitive functions (such as cortical body representations) seem to play a more prominent role $[81,82]$. The iTAD may therefore be especially suited for conditions with altered body representations, such as musculoskeletal disorders [83] and persistent pain [84].

\section{Implications and future directions}


387 Less measurement error for the ITAD could result in better responsiveness, although this needs

388 investigation in future trials studying treatment effects. Further, the multiple measures of the iTAD may

389 provide a more comprehensive evaluation of tactile acuity function. However, validity of the iTAD

390 assessments has not been thoroughly established, precluding inferences about their clinical utility in

391 addition to, or instead of, TPDT. Moreover, their clinical relevance needs further examination in patient

392 trials. Additionally, future studies could explore to what extent iTAD scores reflect central

393 somatosensory processing using neuroimaging techniques. Future studies may also investigate the

394 clinimetric properties and clinical utility of other promising manual [36, 37, 44, 85] and automated [86]

395 procedures, including automated TPDT $[62,87]$, at the neck.

396

397

398 Conclusion

399 Findings suggest that the ITAD and TPDT have similar inter-rater reliability when measuring tactile

400 acuity at the neck in healthy individuals. However, the iTAD exhibits several advantages such as ability to

401 assess multiple aspects of tactile acuity, less measurement error and a possibility for independent

402 sensory training. Furthermore, no evidence was found that scores were affected by anthropometry,

403 simplifying interpretation. Additionally, internal consistency of iTAD accuracy scores was high and no

404 overt floor or ceiling effects were detected. These results highlight the potential clinical utility of the

405 ITAD and support continued investigation. 
406 References

407 [1] J. Debenham, P. Butler, A. Mallows and B.M. Wand, "Disrupted Tactile Acuity in People With Achilles 408 Tendinopathy: A Preliminary Case-Control Investigation," J.Orthop.Sports Phys.Ther., vol. 46, pp. 1061409 1064, Dec. 2016.

410 [2] S. Fujimoto and N. Kon, "Improvement of tactile roughness discrimination acuity correlates with

411 perception of improved hand function in patients after hand surgery," J.Phys.Ther.Sci., vol. 28, pp. 1307412 1311, Apr. 2016.

413 [3] S. Mena-Del Horno, M. Balasch-Bernat, L. Duenas, F. Reis, A. Louw and E. Lluch, "Laterality

414 judgement and tactile acuity in patients with frozen shoulder: A cross-sectional study,"

415 Musculoskelet.Sci.Pract., vol. 47, pp. 102136, Jun. 2020.

416 [4] M.J. Catley, N.E. O'Connell, C. Berryman, F.F. Ayhan and G.L. Moseley, "Is tactile acuity altered in 417 people with chronic pain? a systematic review and meta-analysis," J.Pain, vol. 15, pp. 985-1000, Oct. 4182014.

419 [5] D.S. Harvie, G. Edmond-Hank and A.D. Smith, "Tactile acuity is reduced in people with chronic neck 420 pain," Musculoskelet.Sci.Pract., vol. 33, pp. 61-66, Feb. 2018.

421 [6] F. Vega-Bermudez and K.O. Johnson, "Spatial acuity after digit amputation," Brain, vol. 125, pp. 1256422 1264, Jun. 2002.

423 [7] M. Guemann, S. Bouvier, C. Halgand, F. Paclet, L. Borrini, D. Ricard, E. Lapeyre, D. Cattaert and A. 424 Rugy, "Effect of vibration characteristics and vibror arrangement on the tactile perception of the upper 425 arm in healthy subjects and upper limb amputees," J.Neuroeng Rehabil., vol. 16, pp. 138-019-0597-6, 426 Nov 13. 2019.

427 [8] M.C.R. Fonseca, V.M.C. Elui, E. Lalone, N.C. da Silva, R.I. Barbosa, A.M. Marcolino, F.P.F.M. Ricci and 428 J.C. MacDermid, "Functional, motor, and sensory assessment instruments upon nerve repair in adult 429 hands: systematic review of psychometric properties," Syst.Rev., vol. 7, pp. 175-018-0836-0, Oct 27. 4302018.

431 [9] M.D. Rinderknecht, J.A. Duenas, J.P. Held, O. Lambercy, F.M. Conti, L. Zizlsperger, A.R. Luft, M.C. 432 Hepp-Reymond and R. Gassert, "Automated and Quantitative Assessment of Tactile Mislocalization 433 After Stroke," Front.Neurol., vol. 10, pp. 593, Jun 12. 2019.

434 [10] T.R. Stanton, C.W. Lin, H. Bray, R.J. Smeets, D. Taylor, R.Y. Law and G.L. Moseley, "Tactile acuity is 435 disrupted in osteoarthritis but is unrelated to disruptions in motor imagery performance,"

436 Rheumatology (Oxford), vol. 52, pp. 1509-1519, Aug. 2013. 
437 [11] J.S. Lewis and P. Schweinhardt, "Perceptions of the painful body: the relationship between body 438 perception disturbance, pain and tactile discrimination in complex regional pain syndrome," Eur.J.Pain, 439 vol. 16, pp. 1320-1330, Oct. 2012.

440 [12] G. Zeilig, S. Enosh, D. Rubin-Asher, B. Lehr and R. Defrin, "The nature and course of sensory changes 441 following spinal cord injury: predictive properties and implications on the mechanism of central pain,"

442 Brain, vol. 135, pp. 418-430, Feb. 2012.

443 [13] B. Pleger, M. Tegenthoff, P. Ragert, A.F. Forster, H.R. Dinse, P. Schwenkreis, V. Nicolas and C. Maier, 444 "Sensorimotor retuning [corrected] in complex regional pain syndrome parallels pain reduction,"

445 Ann.Neurol., vol. 57, pp. 425-429, Mar. 2005.

446 [14] L.G. Moseley, N.M. Zalucki and K. Wiech, "Tactile discrimination, but not tactile stimulation alone, 447 reduces chronic limb pain," Pain, vol. 137, pp. 600-608, Jul 31. 2008.

448 [15] A.C. Schmid, A. Schwarz, S.M. Gustin, J.D. Greenspan, F.C. Hummel and N. Birbaumer, "Pain 449 reduction due to novel sensory-motor training in Complex Regional Pain Syndrome I - A pilot study," 450 Scand.J.Pain, vol. 15, pp. 30-37, Apr. 2017.

451 [16] R. Wakolbinger, M. Diers, L.A. Hruby, A. Sturma and O.C. Aszmann, "Home-Based Tactile 452 Discrimination Training Reduces Phantom Limb Pain," Pain Pract., vol. 18, pp. 709-715, Jul. 2018.

453 [17] B.M. Wand, S. Abbaszadeh, A.J. Smith, M.J. Catley and G.L. Moseley, "Acupuncture applied as a 454 sensory discrimination training tool decreases movement-related pain in patients with chronic low back 455 pain more than acupuncture alone: a randomised cross-over experiment," Br.J.Sports Med., vol. 47, pp. 456 1085-1089, Nov. 2013.

457 [18] E. Walter, Cambridge advanced learner's dictionary, Cambridge university press, 2008, .

458 [19] D. Marsh, "The validation of measures of outcome following suture of divided peripheral nerves 459 supplying the hand," J.Hand Surg.Br., vol. 15, pp. 25-34, Feb. 1990.

460 [20] J. Bell-Krotoski, S. Weinstein and C. Weinstein, "Testing sensibility, including touch-pressure, two461 point discrimination, point localization, and vibration," J.Hand Ther., vol. 6, pp. 114-123, Apr-Jun. 1993.

462 [21] J.C. Stevens and M.Q. Patterson, "Dimensions of spatial acuity in the touch sense: changes over the 463 life span," Somatosens.Mot.Res., vol. 12, pp. 29-47, 1995.

464 [22] V.E. Abraira and D.D. Ginty, "The sensory neurons of touch," Neuron, vol. 79, pp. 618-639, Aug 21. 4652013.

466 [23] S. Demain, C.D. Metcalf, G.V. Merrett, D. Zheng and S. Cunningham, "A narrative review on haptic 467 devices: relating the physiology and psychophysical properties of the hand to devices for rehabilitation 468 in central nervous system disorders," Disabil.Rehabil.Assist.Technol., vol. 8, pp. 181-189, May. 2013. 
469 [24] K. Ehrenbrusthoff, C.G. Ryan, C. Gruneberg and D.J. Martin, "A systematic review and meta-analysis 470 of the reliability and validity of sensorimotor measurement instruments in people with chronic low back 471 pain," Musculoskelet.Sci.Pract., vol. 35, pp. 73-83, Jun. 2018.

472 [25] R. Boldt, J. Gogulski, J. Guzman-Lopez, S. Carlson and A. Pertovaara, "Two-point tactile

473 discrimination ability is influenced by temporal features of stimulation," Exp.Brain Res., vol. 232, pp.

474 2179-2185, Jul. 2014.

475 [26] J.C. Craig and K.O. Johnson, "The Two-Point Threshold: Not a Measure of Tactile Spatial Resolution," 476 Curr Dir Psychol Sci, vol. 9, pp. 29-32, 02/01; 2017/10. 2000.

477 [27] G. Lundborg and B. Rosen, "The two-point discrimination test--time for a re-appraisal?" J.Hand 478 Surg.Br., vol. 29, pp. 418-422, Oct. 2004.

479 [28] J. Tong, O. Mao and D. Goldreich, "Two-point orientation discrimination versus the traditional two480 point test for tactile spatial acuity assessment," Front.Hum.Neurosci., vol. 7, pp. 579, Sep 13. 2013.

481 [29] T. Kalisch, P. Ragert, P. Schwenkreis, H.R. Dinse and M. Tegenthoff, "Impaired tactile acuity in old 482 age is accompanied by enlarged hand representations in somatosensory cortex," Cereb.Cortex, vol. 19, 483 pp. 1530-1538, Jul. 2009.

484 [30] T. Kalisch, J.C. Kattenstroth, R. Kowalewski, M. Tegenthoff and H.R. Dinse, "Cognitive and tactile 485 factors affecting human haptic performance in later life," PLoS One, vol. 7, pp. e30420, 2012.

486 [31] C. Falling and R. Mani, "Regional asymmetry, obesity and gender determines tactile acuity of the 487 knee regions: A cross-sectional study," Man.Ther., vol. 26, pp. 150-157, Dec. 2016.

488 [32] R.M. Peters, E. Hackeman and D. Goldreich, "Diminutive digits discern delicate details: fingertip size 489 and the sex difference in tactile spatial acuity," J.Neurosci., vol. 29, pp. 15756-15761, Dec 16. 2009.

490 [33] M.J. Catley, A. Tabor, B.M. Wand and G.L. Moseley, "Assessing tactile acuity in rheumatology and 491 musculoskeletal medicine--how reliable are two-point discrimination tests at the neck, hand, back and 492 foot?" Rheumatology (Oxford), vol. 52, pp. 1454-1461, Aug. 2013.

493 [34] C. Jerosch-Herold, "A study of the relative responsiveness of five sensibility tests for assessment of 494 recovery after median nerve injury and repair," J.Hand Surg.Br., vol. 28, pp. 255-260, Jun. 2003.

495 [35] W. Adamczyk, A. Slugocka, O. Saulicz and E. Saulicz, "The point-to-point test: A new diagnostic tool 496 for measuring lumbar tactile acuity? Inter and intra-examiner reliability study of pain-free subjects," 497 Man.Ther., vol. 22, pp. 220-226, Apr. 2016.

498 [36] P. Bruns, C.J. Camargo, H. Campanella, J. Esteve, H.R. Dinse and B. Roder, "Tactile acuity charts: a 499 reliable measure of spatial acuity," PLoS One, vol. 9, pp. e87384, Feb 4. 2014. 
500 [37] R.W. Van Boven, R.H. Hamilton, T. Kauffman, J.P. Keenan and A. Pascual-Leone, "Tactile spatial

501 resolution in blind braille readers," Neurology, vol. 54, pp. 2230-2236, Jun 27. 2000.

502 [38] W.M. Adamczyk, A. Slugocka, K. Mehlich, E. Saulicz and K. Luedtke, "Preliminary Validation of a

503 Two-Point Estimation Task for the Measurement of Sensory Dissociation in Patients with Chronic Low

504 Back Pain," Pain Med., vol. 20, pp. 2472-2478, Dec 1. 2019.

505 [39] K. Zimney, G. Dendinger, M. Engel and J. Mitzel, "Comparison of reliability and efficiency of two

506 modified two-point discrimination tests and two-point estimation tactile acuity test," Physiother.Theory

507 Pract., pp. 1-10, Jan 29. 2020.

508 [40] R. Hoffmann, V.V. Valgeirsdottir, O.I. Johannesson, R. Unnthorsson and A. Kristjansson, "Measuring

509 relative vibrotactile spatial acuity: effects of tactor type, anchor points and tactile anisotropy," Exp.Brain

510 Res., vol. 236, pp. 3405-3416, Dec. 2018.

511 [41] C. Ryan, N. Harland, B.T. Drew and D. Martin, "Tactile acuity training for patients with chronic low

512 back pain: a pilot randomised controlled trial," BMC Musculoskelet.Disord., vol. 15, pp. 59-2474-15-59,

513 Feb 26. 2014.

514 [42] Global Burden of Disease Study 2013 Collaborators, "Global, regional, and national incidence,

515 prevalence, and years lived with disability for 301 acute and chronic diseases and injuries in 188

516 countries, 1990-2013: a systematic analysis for the Global Burden of Disease Study 2013," Lancet, vol.

517 386, pp. 743-800, Aug 22. 2015.

518 [43] D.S. Harvie, J. Kelly, H. Buckman, J. Chan, G. Sutherland, M. Catley, J. Novak, N. Tuttle and M.

519 Sterling, "Tactile acuity testing at the neck: A comparison of methods," Musculoskelet.Sci.Pract., vol. 32, 520 pp. 23-30, Dec. 2017.

521 [44] K.A. Morrow and M. Ziat, "Tactile spatial acuity of the neck using a two-point orientation

522 discrimination task," in - 2018 IEEE Haptics Symposium (HAPTICS), pp. 296-300, 2018.

523 [45] W.M. Adamczyk, A. Budzisz, O. Saulicz, T.M. Szikszay, E. Saulicz and K. Luedtke, "Tactile Precision

524 Remains Intact When Acute Neck Pain Is Induced," J.Pain, vol. 20, pp. 1070-1079, Sep. 2019.

525 [46] K. Luedtke and W. Adamczyk, "Tactile acuity in the neck: calling for more basic science research,"

526 Musculoskelet.Sci.Pract., vol. 32, pp. 127-128, Dec. 2017.

527 [47] N.A. Olthof, D.S. Harvie, C. Henderson, B. Thompson, R. Sharp, L. Craig-Ward, J.D. Weermeijer, M.

528 Sterling, G.L. Moseley and M.W. Coppieters, "Description and psychometric properties of a prototype to 529 test tactile acuity in the neck," Musculoskelet.Sci.Pract., vol. 51, pp. 102259, Feb. 2021. 
530 [48] K. Luedtke, W. Adamczyk, K. Mehrtens, I. Moeller, L. Rosenbaum, A. Schaefer, J. Schroeder, T.

531 Szikszay, C. Zimmer and B. Wollesen, "Upper cervical two-point discrimination thresholds in migraine

532 patients and headache-free controls," J.Headache Pain, vol. 19, pp. 47-018-0873-z, Jun 26. 2018.

533 [49] A. Vandierendonck, "A comparison of methods to combine speed and accuracy measures of

534 performance: A rejoinder on the binning procedure," Behav.Res.Methods, vol. 49, pp. 653-673, Apr.

5352017.

536 [50] L.G. Portney and M.P. Watkins, Foundations of clinical research: applications to practice,

537 Pearson/Prentice Hall Upper Saddle River, NJ, 2009, .

538 [51] H.C. de Vet, C.B. Terwee, D.L. Knol and L.M. Bouter, "When to use agreement versus reliability

539 measures," J.Clin.Epidemiol., vol. 59, pp. 1033-1039, Oct. 2006.

540 [52] W.G. Hopkins, "Measures of reliability in sports medicine and science," Sports Med., vol. 30, pp. 1-

541 15, Jul. 2000.

542 [53] G. Atkinson and A.M. Nevill, "Statistical methods for assessing measurement error (reliability) in

543 variables relevant to sports medicine," Sports Med., vol. 26, pp. 217-238, Oct. 1998.

544 [54] H. Quan and W.J. Shih, "Assessing reproducibility by the within-subject coefficient of variation with 545 random effects models," Biometrics, vol. 52, pp. 1195-1203, Dec. 1996.

546 [55] S.D. Walter, M. Eliasziw and A. Donner, "Sample size and optimal designs for reliability studies,"

547 Stat.Med., vol. 17, pp. 101-110, Jan 15. 1998.

548 [56] K.S. Taber, "The Use of Cronbach's Alpha When Developing and Reporting Research Instruments in 549 Science Education," Res Sci Educ, vol. 48, pp. 1273-1296, 12/01. 2018.

550 [57] C.B. Terwee, S.D. Bot, M.R. de Boer, D.A. van der Windt, D.L. Knol, J. Dekker, L.M. Bouter and H.C.

551 de Vet, "Quality criteria were proposed for measurement properties of health status questionnaires,"

552 J.Clin.Epidemiol., vol. 60, pp. 34-42, Jan. 2007.

553 [58] H.Y. Kim, "Statistical notes for clinical researchers: assessing normal distribution (2) using skewness 554 and kurtosis," Restor.Dent.Endod., vol. 38, pp. 52-54, Feb. 2013.

555 [59] A.D. Ho and C.C. Yu, "Descriptive Statistics for Modern Test Score Distributions: Skewness, Kurtosis,

556 Discreteness, and Ceiling Effects," Educ.Psychol.Meas., vol. 75, pp. 365-388, Jun. 2015.

557 [60] W.M. Adamczyk, K. Luedtke and T.M. Szikszay, "Two-point discrimination and the low back pain:

558 Not as unreliable as it seems, but what about standardised procedures?" Musculoskelet.Sci.Pract., vol.

559 35, pp. e110-e111, Jun. 2018.

560 [61] A.G. Cashin, "Measuring two-point discrimination threshold with a caliper," J.Physiother., vol. 63,

561 pp. 186, Jul. 2017.

Peer] reviewing PDF | (2021:05:60922:1:0:NEW 28 Jul 2021) 
562 [62] H. Yokota, N. Otsuru, R. Kikuchi, R. Suzuki, S. Kojima, K. Saito, S. Miyaguchi, Y. Inukai and H. Onishi,

563 "Establishment of optimal two-point discrimination test method and consideration of reproducibility,"

564 Neurosci.Lett., vol. 714, pp. 134525, Jan 1. 2020.

565 [63] K.M. Cheever, J.W. Myrer, A.W. Johnson and G.W. Fellingham, "Understanding the complete

566 pathophysiology of chronic mild to moderate neck pain: Implications for the inclusion of a

567 comprehensive sensorimotor evaluation," J.Back Musculoskelet.Rehabil., vol. 30, pp. 991-997, Sep 22.

5682017.

569 [64] S. Elsig, H. Luomajoki, M. Sattelmayer, J. Taeymans, A. Tal-Akabi and R. Hilfiker, "Sensorimotor tests, 570 such as movement control and laterality judgment accuracy, in persons with recurrent neck pain and

571 controls. A case-control study," Man.Ther., vol. 19, pp. 555-561, Dec. 2014.

572 [65] S.B. Green, Y. Yang, M. Alt, S. Brinkley, S. Gray, T. Hogan and N. Cowan, "Use of internal consistency 573 coefficients for estimating reliability of experimental task scores," Psychon.Bull.Rev., vol. 23, pp. 750-

574763 , Jun. 2016.

575 [66] G.J. Matheson, "We need to talk about reliability: making better use of test-retest studies for study

576 design and interpretation," PeerJ, vol. 7, pp. e6918, May 24. 2019.

577 [67] R. van Lien, M. Neijts, G. Willemsen and E.J. de Geus, "Ambulatory measurement of the ECG T-wave 578 amplitude," Psychophysiology, vol. 52, pp. 225-237, Feb. 2015.

579 [68] D.N. Towers and J.J. Allen, "A better estimate of the internal consistency reliability of frontal EEG

580 asymmetry scores," Psychophysiology, vol. 46, pp. 132-142, Jan. 2009.

581 [69] A. Domingo and T. Lam, "Reliability and validity of using the Lokomat to assess lower limb joint 582 position sense in people with incomplete spinal cord injury," J.Neuroeng Rehabil., vol. 11, pp. 167-0003583 11-167, Dec 16. 2014.

584 [70] T. Platz, K. Prass, P. Denzler, S. Bock and K.H. Mauritz, "Testing a motor performance series and a 585 kinematic motion analysis as measures of performance in high-functioning stroke patients: reliability, 586 validity, and responsiveness to therapeutic intervention," Arch.Phys.Med.Rehabil., vol. 80, pp. 270-277, 587 Mar. 1999.

588 [71] C. Falling and R. Mani, "Ageing and obesity indices influences the tactile acuity of the low back 589 regions: A cross-sectional study," Man.Ther., vol. 23, pp. 25-31, Jun. 2016.

590 [72] M. Lenz, M. Tegenthoff, K. Kohlhaas, P. Stude, O. Hoffken, M.A. Gatica Tossi, T. Kalisch, R.

591 Kowalewski and H.R. Dinse, "Increased excitability of somatosensory cortex in aged humans is

592 associated with impaired tactile acuity," J.Neurosci., vol. 32, pp. 1811-1816, Feb 1. 2012.

Peer] reviewing PDF | (2021:05:60922:1:0:NEW 28 Jul 2021) 
593 [73] S. Brodoehl, C. Klingner, K. Stieglitz and O.W. Witte, "Age-related changes in the somatosensory

594 processing of tactile stimulation--an fMRI study," Behav.Brain Res., vol. 238, pp. 259-264, Feb 1. 2013.

595 [74] B. Pleger, C. Wilimzig, V. Nicolas, T. Kalisch, P. Ragert, M. Tegenthoff and H.R. Dinse, "A

596 complementary role of intracortical inhibition in age-related tactile degradation and its remodelling in

597 humans," Sci.Rep., vol. 6, pp. 27388, Jun 15. 2016.

598 [75] H.R. Dinse, N. Kleibel, T. Kalisch, P. Ragert, C. Wilimzig and M. Tegenthoff, "Tactile coactivation

599 resets age-related decline of human tactile discrimination," Ann.Neurol., vol. 60, pp. 88-94, Jul. 2006.

600 [76] C. Falling and R. Mani, "(436) Influence of demographic and anthropometric factors on tactile acuity

601 of various body regions: a systematic narrative review," J. Pain, vol. 18, pp. s83, 2017.

602 [77] S.Y. Won, H.K. Kim, M.E. Kim and K.S. Kim, "Two-point discrimination values vary depending on test 603 site, sex and test modality in the orofacial region: a preliminary study," J.Appl.Oral Sci., vol. 25, pp. 427604 435, Jul-Aug. 2017.

605 [78] D.B. Boles and S.M. Givens, "Laterality and sex differences in tactile detection and two-point 606 thresholds modified by body surface area and body fat ratio," Somatosens.Mot.Res., vol. 28, pp. 102607 109, 2011.

608 [79] L.K. Smalls, R. Randall Wickett and M.O. Visscher, "Effect of dermal thickness, tissue composition, 609 and body site on skin biomechanical properties," Skin Res.Technol., vol. 12, pp. 43-49, Feb. 2006.

610 [80] C. Braun, E. Eisele, A. Wuhle, M.C. Stuttgen, C. Schwarz and G. Demarchi, "Mislocalization of near-

611 threshold tactile stimuli in humans: a central or peripheral phenomenon?" Eur.J.Neurosci., vol. 33, pp.

612 499-508, Feb. 2011.

613 [81] M.R. Longo, E. Azanon and P. Haggard, "More than skin deep: body representation beyond primary 614 somatosensory cortex," Neuropsychologia, vol. 48, pp. 655-668, Feb. 2010.

615 [82] L. Tame, E. Azanon and M.R. Longo, "A Conceptual Model of Tactile Processing across Body 616 Features of Size, Shape, Side, and Spatial Location," Front.Psychol., vol. 10, pp. 291, Feb 26. 2019.

617 [83] A. Viceconti, E.M. Camerone, D. Luzzi, D. Pentassuglia, M. Pardini, D. Ristori, G. Rossettini, A.

618 Gallace, M.R. Longo and M. Testa, "Explicit and Implicit Own's Body and Space Perception in Painful

619 Musculoskeletal Disorders and Rheumatic Diseases: A Systematic Scoping Review,"

620 Front.Hum.Neurosci., vol. 14, pp. 83, Apr 9. 2020.

621 [84] A. Tsay, T.J. Allen, U. Proske and M.J. Giummarra, "Sensing the body in chronic pain: a review of 622 psychophysical studies implicating altered body representation," Neurosci.Biobehav.Rev., vol. 52, pp. 623 221-232, May. 2015. 
624 [85] Y. Bleyenheuft and J. Thonnard, "Tactile spatial resolution measured manually: A validation study," 625 Somatosens.Mot.Res., vol. 24, pp. 111-114, 01/01. 2007.

626 [86] D. Goldreich, M. Wong, R.M. Peters and I.M. Kanics, "A Tactile Automated Passive-Finger Stimulator 627 (TAPS)," J.Vis.Exp., vol. (28). pii: 1374. doi, pp. 10.3791/1374, Jun 3. 2009.

628 [87] K.S. Frahm and S. Gervasio, "The two-point discrimination threshold depends both on the 629 stimulation noxiousness and modality," Exp.Brain Res., vol. 239, pp. 1439-1449, May. 2021. 
630

631

632

633

635

636

637

638
Figure 1
634

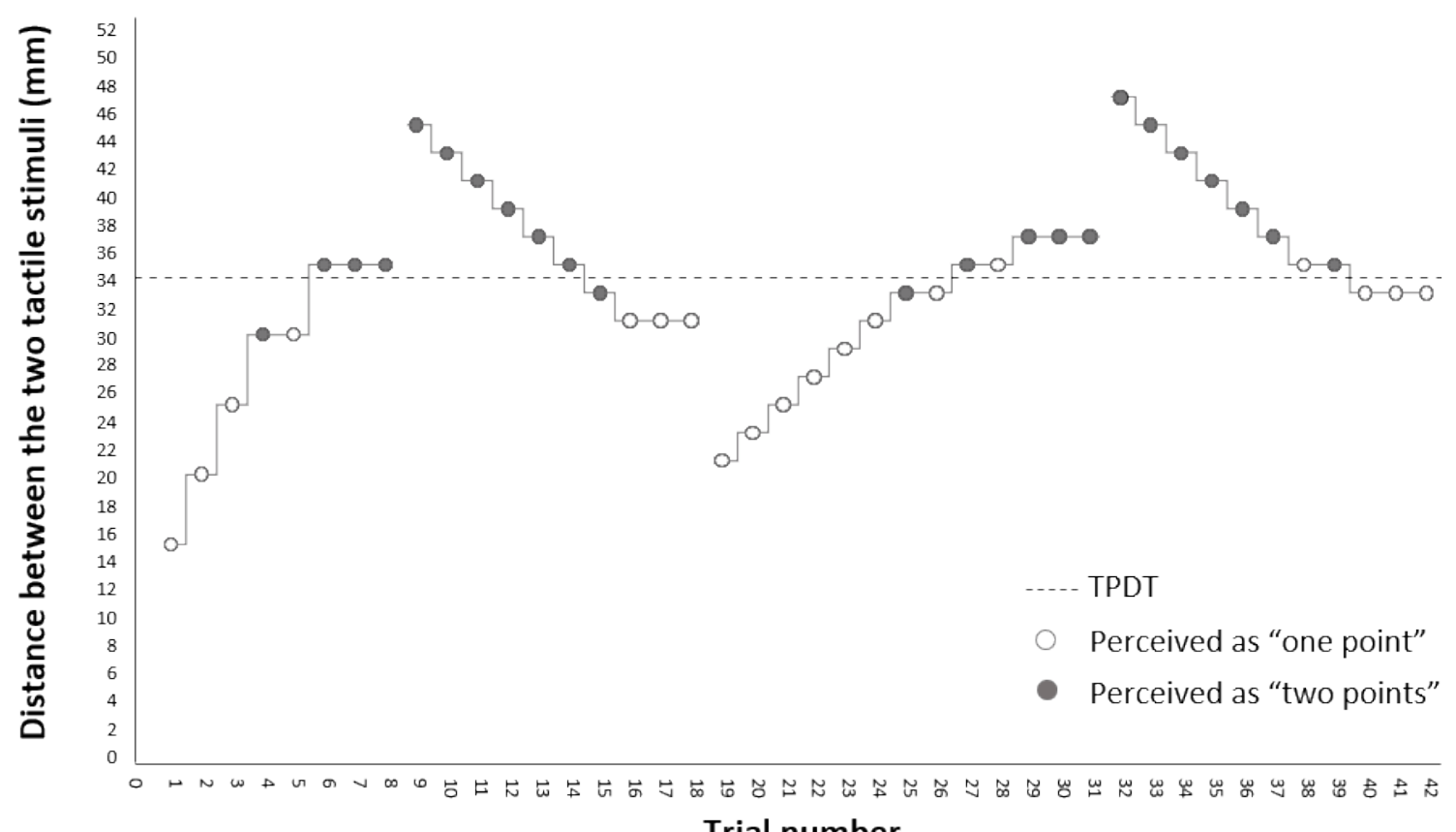

639

640

641

642

643

644

645

Trial number

\section{Figure 1, TPDT assessment procedure}

Example of the assessment of a hypothetical two-point discrimination threshold (TPDT). Assessment is based on a forced-choice response (one or two points), alternating four runs with either increasing or decreasing caliper distances. Steps taken are either in $5 \mathrm{~mm}$. (first run) or $2 \mathrm{~mm}$. (other runs). Three consecutive reports of either one or two points indicates a reversal. Mean of the four reversals is calculated for the TPDT score. 
646

647

648

649

650

651

652

653

654

655

656

657

658

659

660

661

662

663
Figure 2 
665

A.

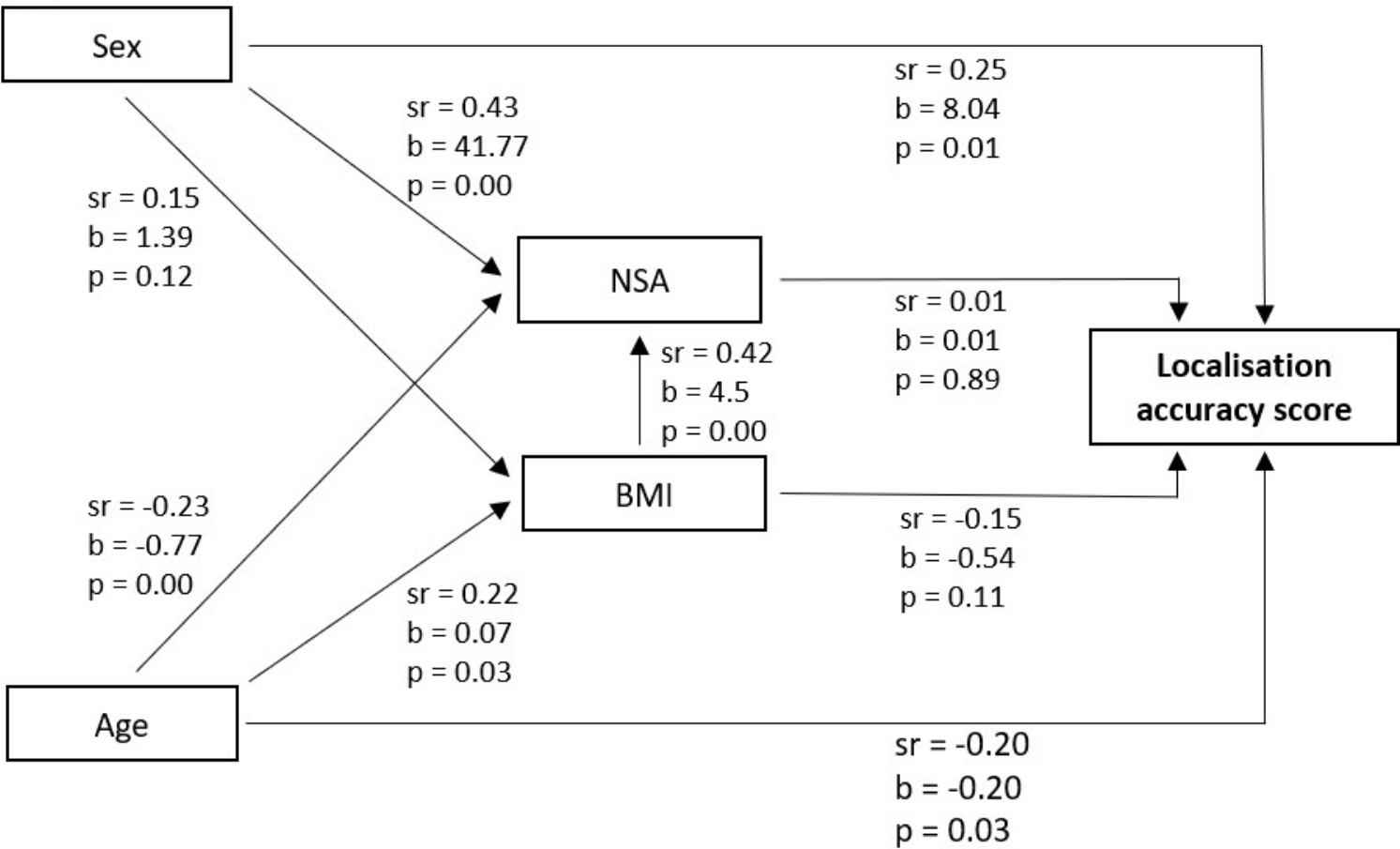

B.

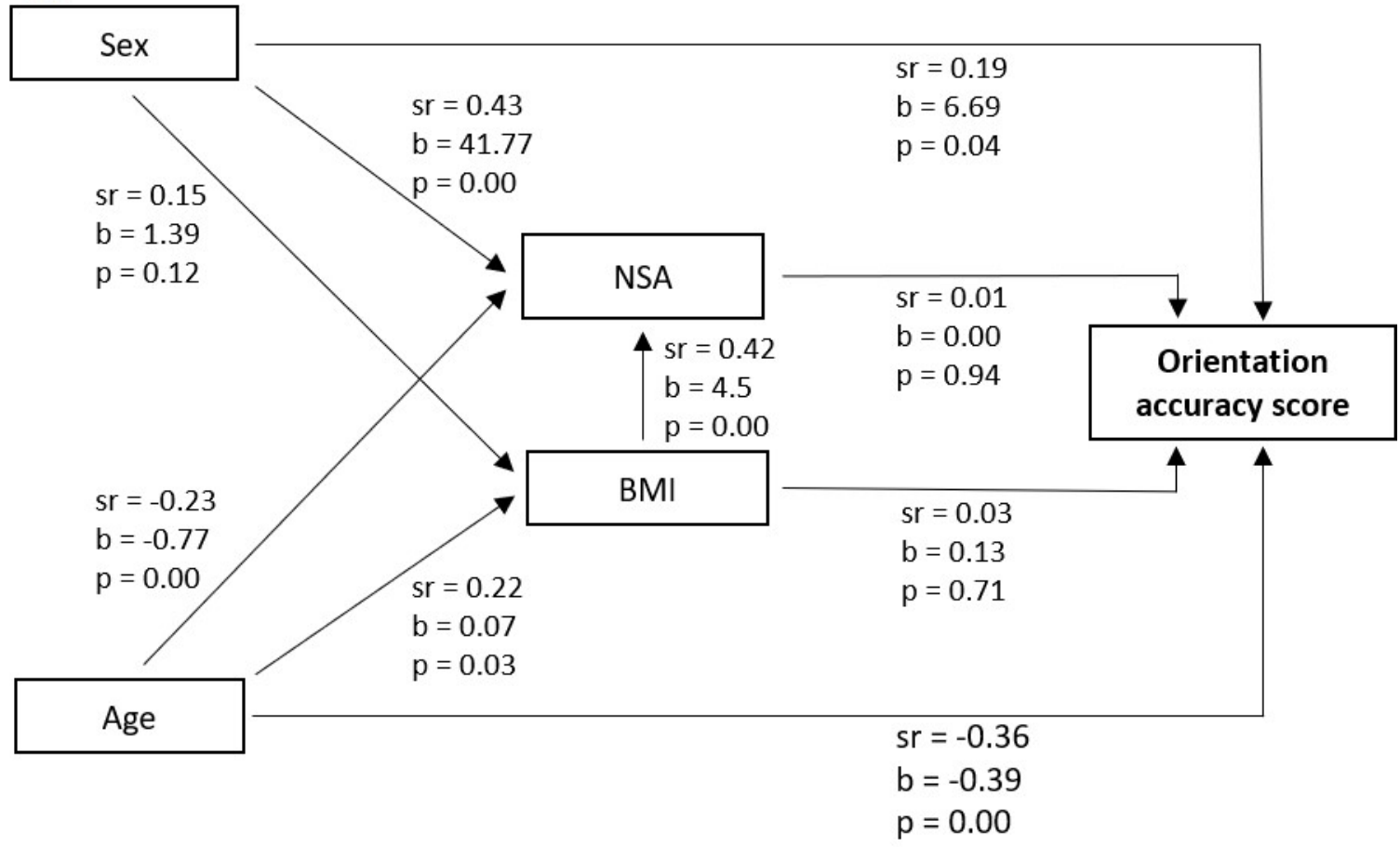

666

667

668 Figure 3, results sequential mediation analyses 
669 Relationships between demographics (sex and age), anthropometrics (body mass index (BMI) and neck 670 surface area (NSA)) and ITAD accuracy scores for the localisation test (Panel A) and orientation test 671 (Panel B). Relationships are expressed in semi-partial correlations (sr) and unstandardized regression 672 coefficients (b), including their level of significance ( $p$ ). Coding for sex: female=0 and male=1. 
673

674

675

676

677

678

679

680

681

682

683

684

685

686

687

688

689

690

691

692

693

694

695

696

697

698

699
Figure 4

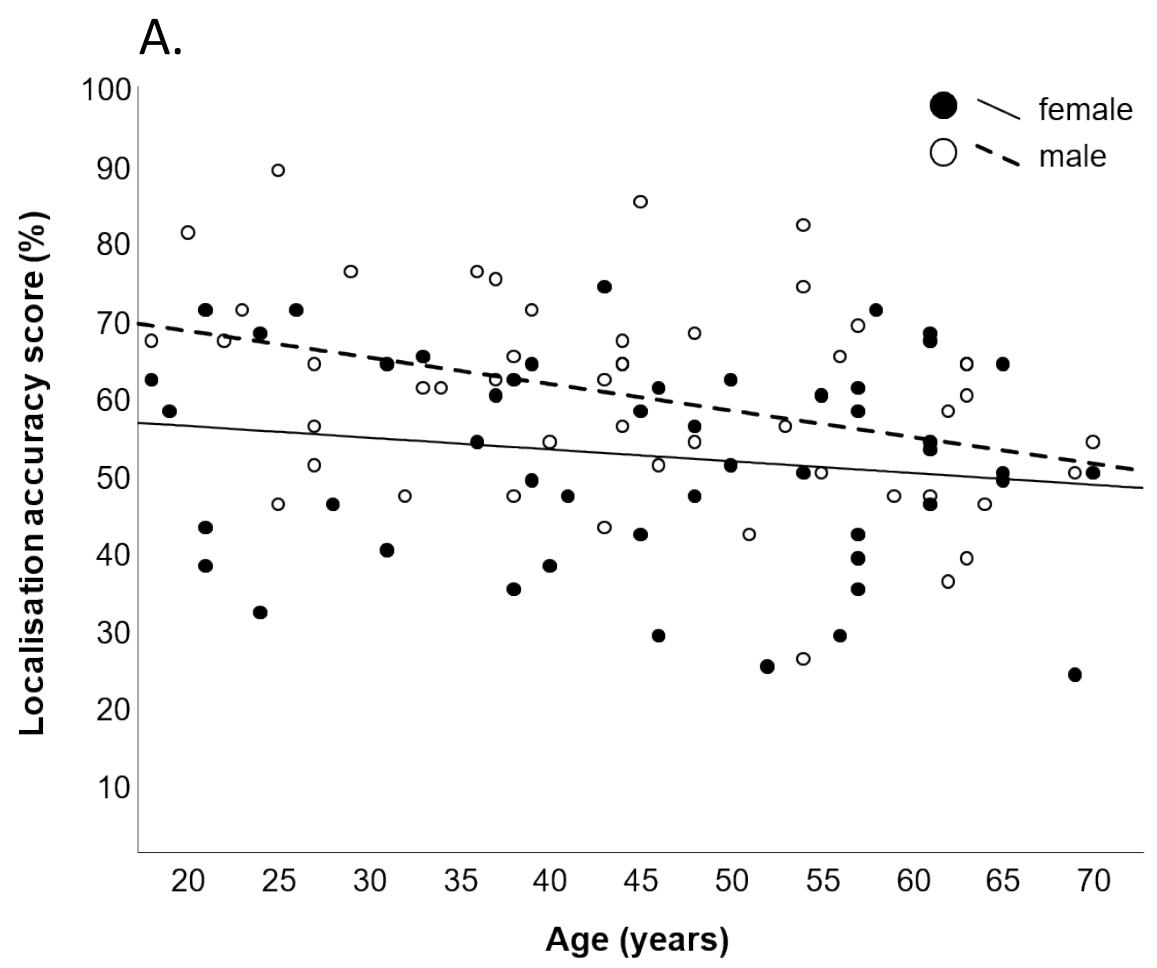

B.

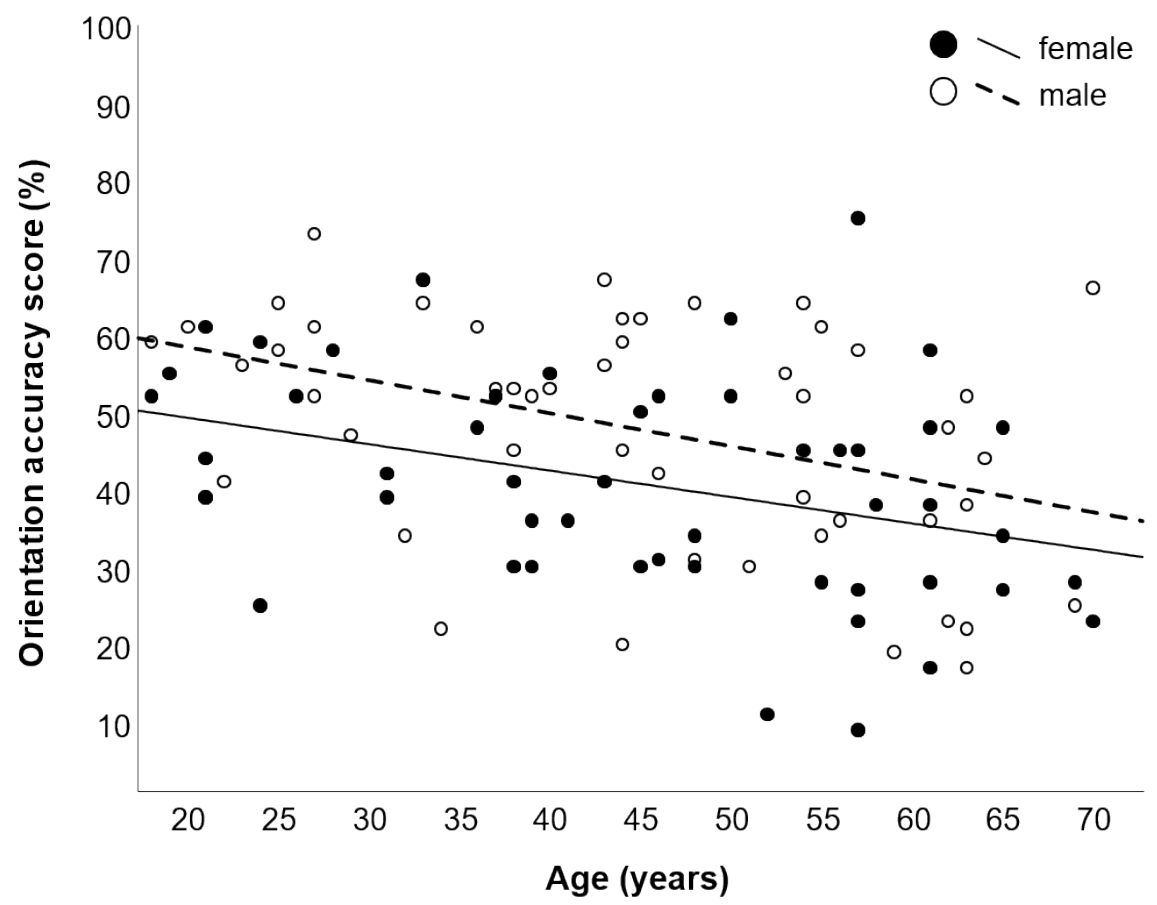

700 Figure 4, scatter plots of localisation and orientation accuracy scores

701 Scatter plots of iTAD accuracy scores as a function of age and sex. Scores are displayed for the 702 localisation (Panel A) and orientation (Panel B) test. Lines represent the least squares regressions. 
703

704

705

706

Table 1

Mean, inter-rater reliability and measurement error for the ITAD and two-point discrimination threshold scores.

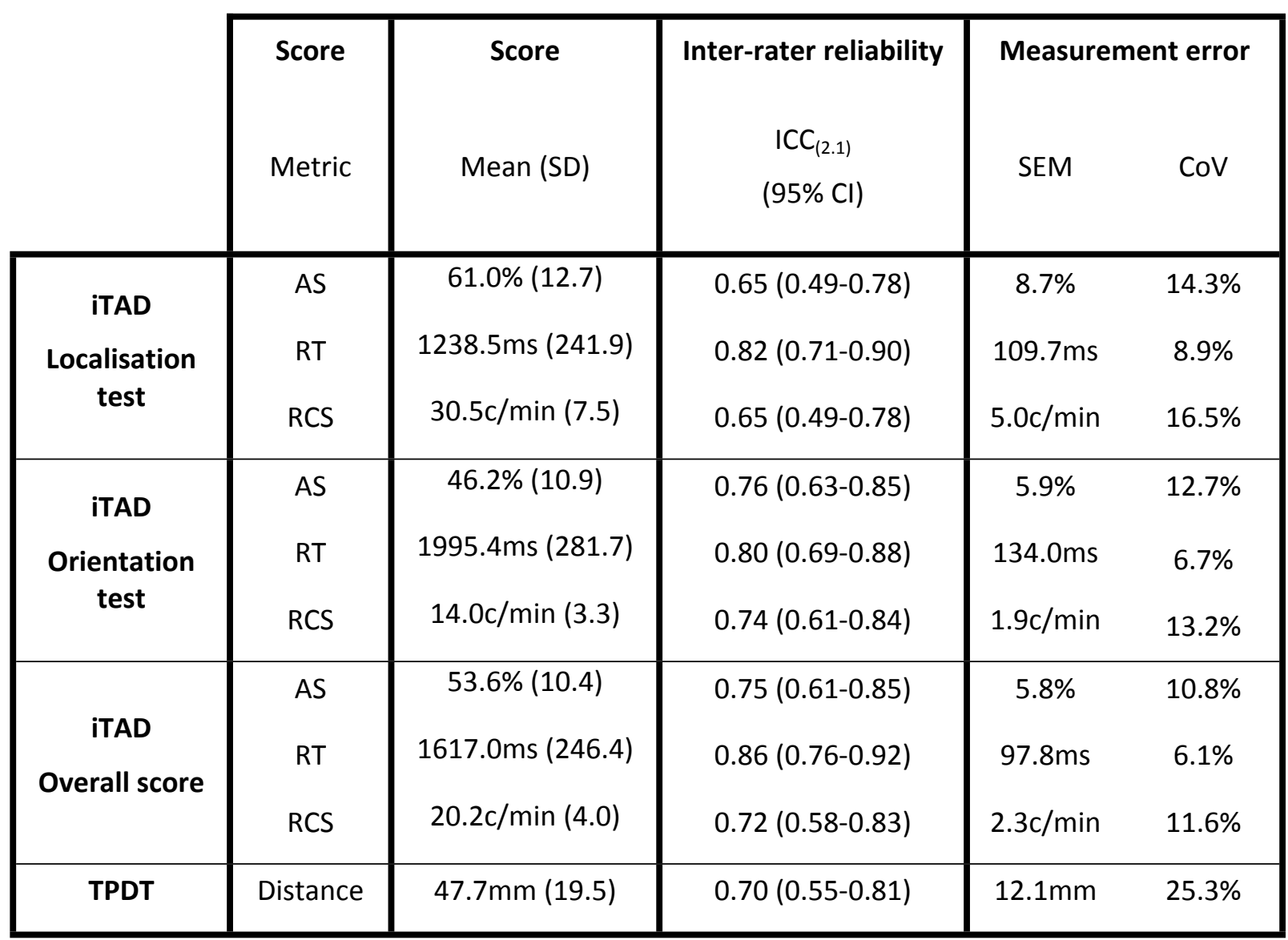

707

708

709

710
ITAD: imprint Tactile Acuity Device; TPDT: two-point discrimination threshold; AS: accuracy score; RT: average response time; RCS: rate correct score; ms: milliseconds; c/min: correct responses per minute; ICC: intraclass correlation coefficient; SEM: standard error of measurement; CoV: coefficient of variation 
711 Table 2

712 Smallest detectable changes with $80 \%, 85 \%, 90 \%$ and $95 \%$ confidence intervals for the iTAD and two-

713 point discrimination threshold scores.

714

715

716

717

718

719

720

721

722

723

724

725

726

727

728

729

730

731

732

733

734

735

ITAD: imprint Tactile Acuity Device; TPDT: two-point discrimination threshold; AS: accuracy score; RT:

736 average response time; RCS: rate correct score; ms: milliseconds; c/min: correct responses per minute;

737 SDC: smallest detectable change 
A.

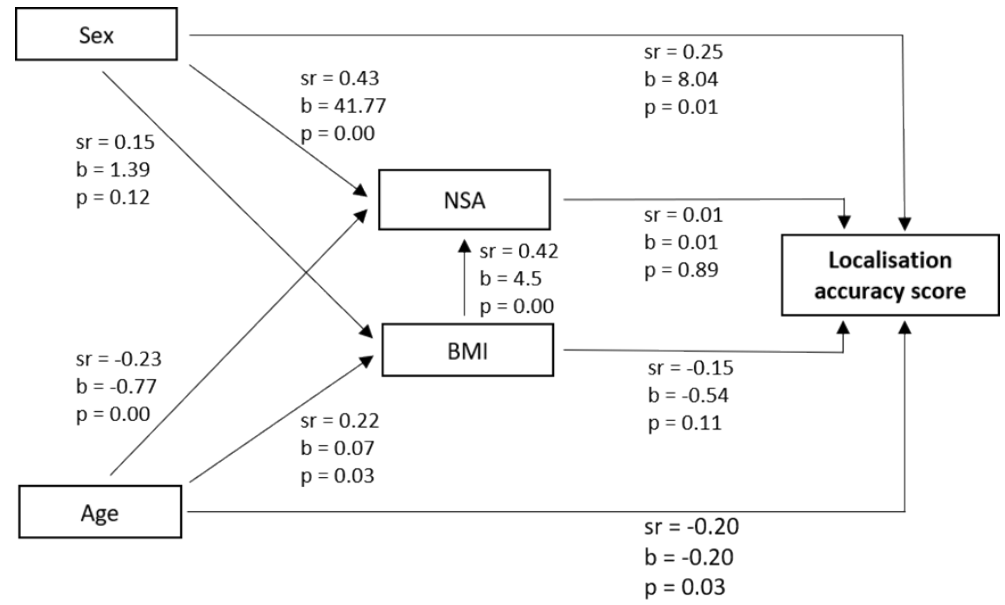

740

B.

742

743

744

745

746

747

748

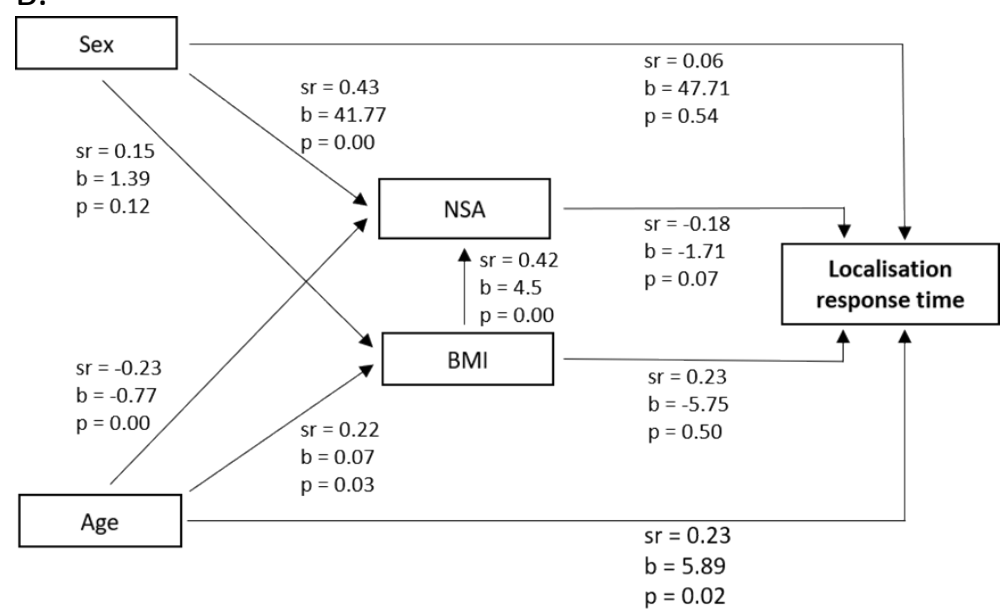

C.

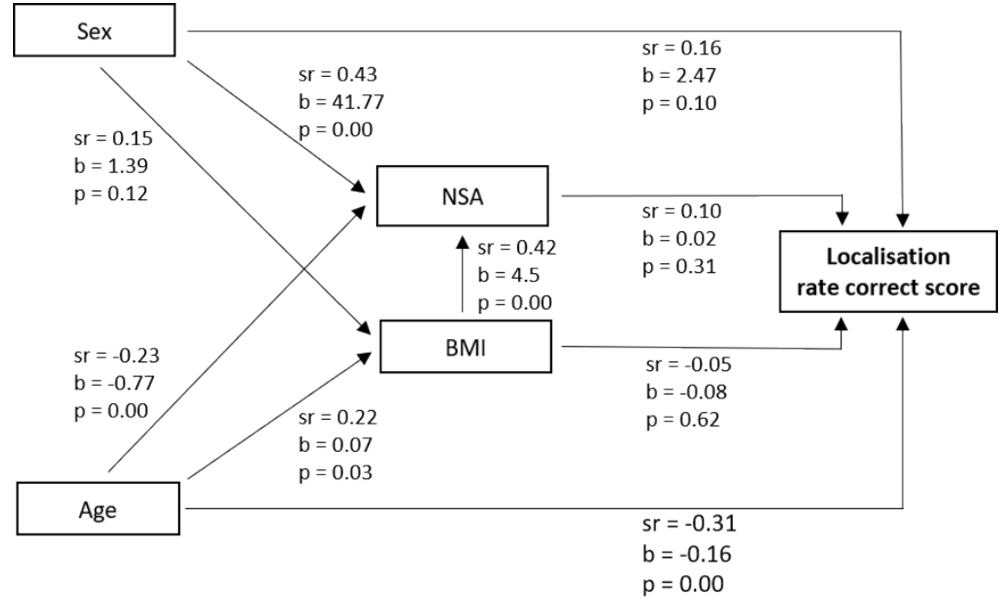

\section{Supplement 1, results sequential mediation analyses iTAD localisation test}

Relationships between age, sex (female=0; male=1), body mass index (BMI), neck surface area (NSA) and iTAD localisation scores from the sequential mediation analyses. Relationships are expressed in semi- 
749 partial correlations ( $\mathrm{sr}$ ) and unstandardized regression coefficients (b), including their level of 750 significance ( $p)$. In all models, BMI and NSA did not significantly ( $p>0.05)$ mediate the relationship 751 between either age or sex with iTAD scores. Models provided for: localisation accuracy score (Panel A), 752 response time (Panel B) and rate correct score (Panel C). 
A.

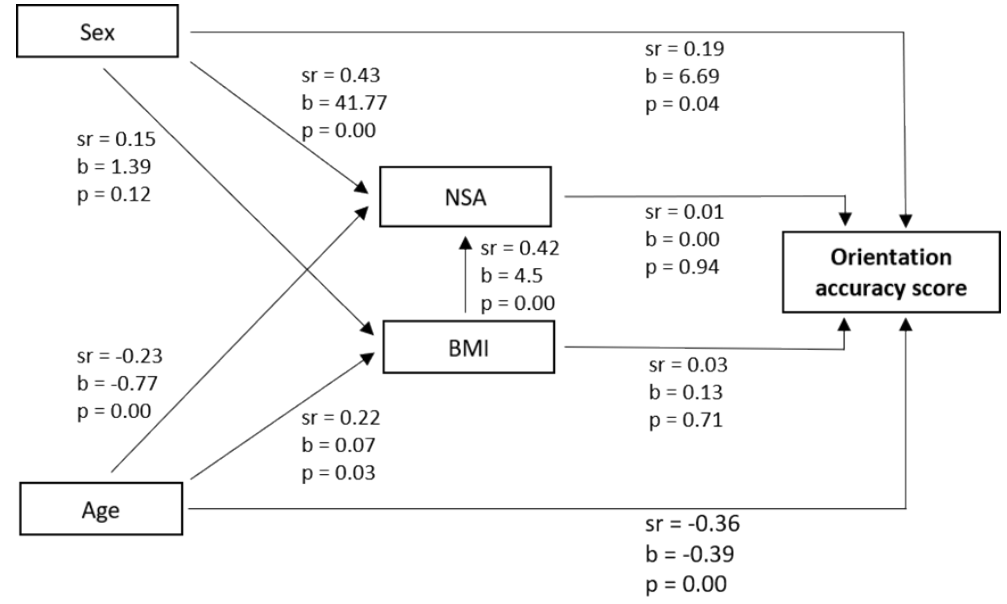

755

B.

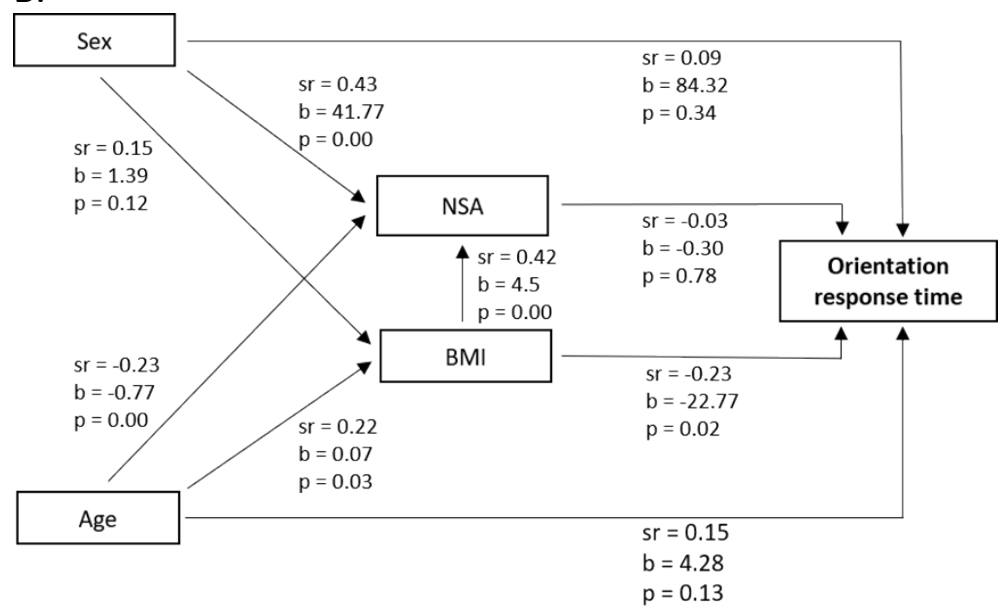

758 C.

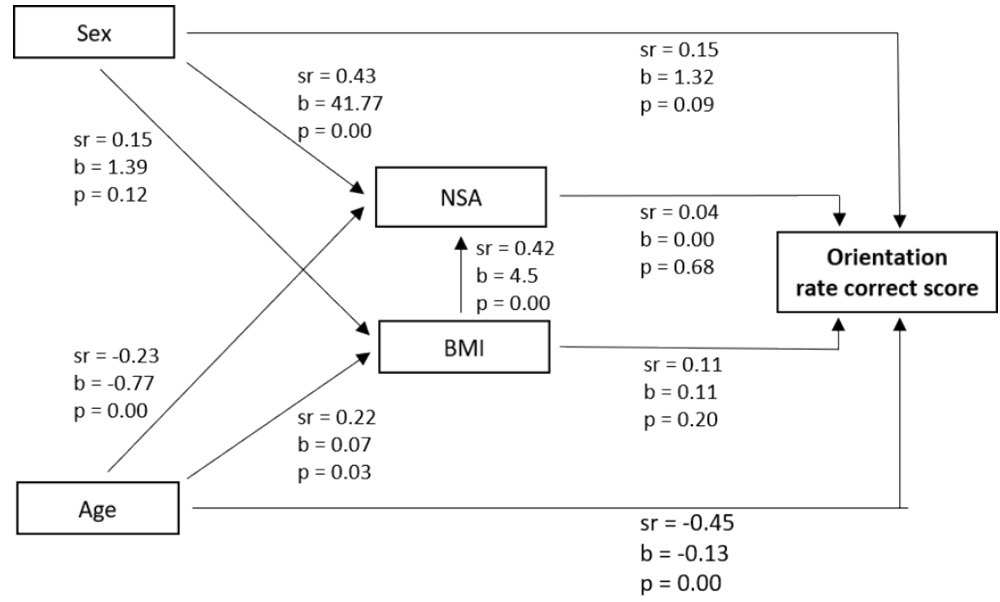


764 partial correlations (sr) and unstandardized regression coefficients (b), including their level of

765 significance $(p)$. In all models, BMI and NSA did not significantly $(p>0.05)$ mediate the relationship

766 between either age or sex with iTAD scores. Models provided for: orientation accuracy score (Panel A),

767 response time (Panel B) and rate correct score (Panel C). 
A.

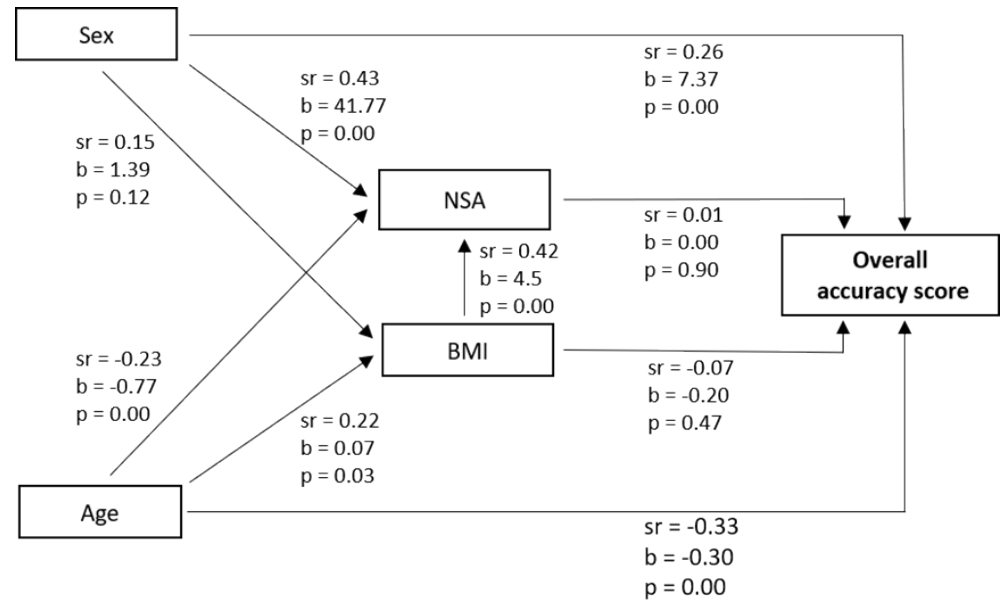

770

771

B.

772

773

774

775

776

777

778

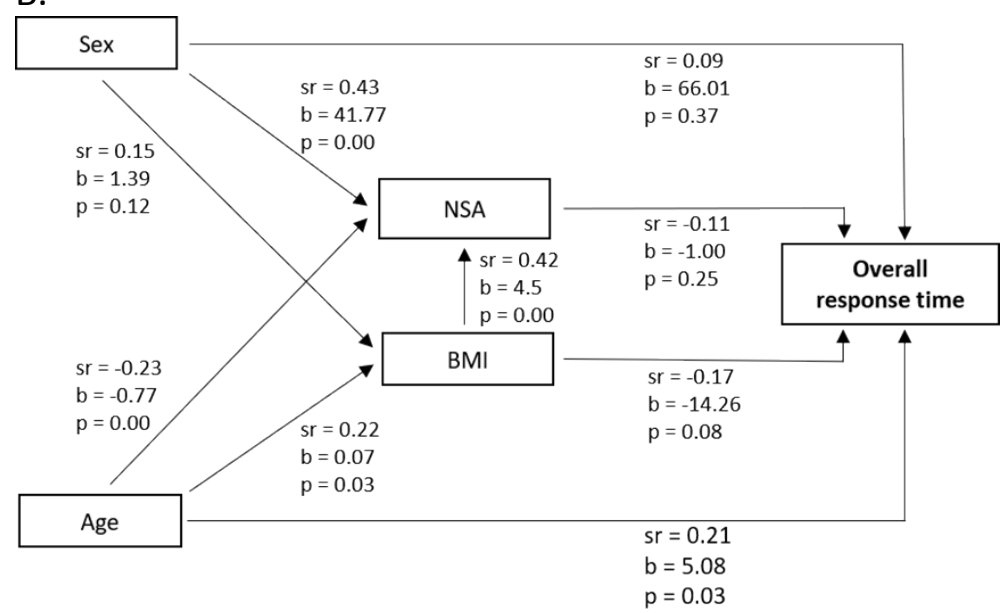

C.

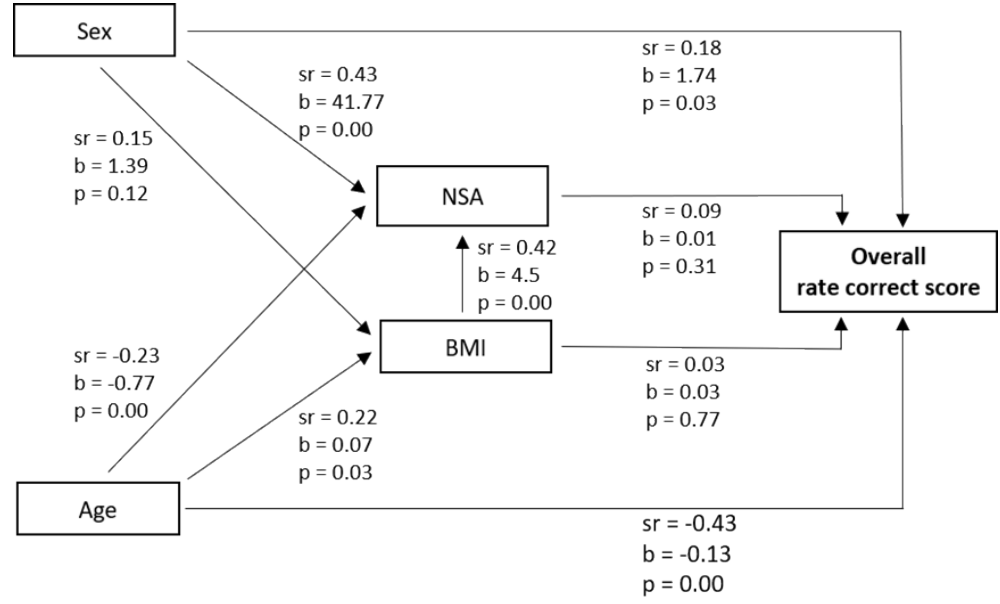

Supplement 3, results sequential mediation analyses iTAD overall score Relationships between age, sex (female=0; male=1), body mass index (BMI), neck surface area (NSA) and iTAD overall scores from the sequential mediation analyses. Relationships are expressed in semi-partial 
779 correlations (sr) and unstandardized regression coefficients (b), including their level of significance (p). In

780 all models, BMI and NSA did not significantly ( $p>0.05)$ mediate the relationship between either age or

781 sex with iTAD scores. Models provided for: overall accuracy score (Panel A), response time (Panel B) and

782 rate correct score (Panel C).

783

784

785

786

787

788

789

790

791

792

793

794

795

796

797

798

799

800

801

802

803

804

805

806

807

808

809

810

811

812

813

814

815

816

817

818

819

820

821

822

823

824

825
A.

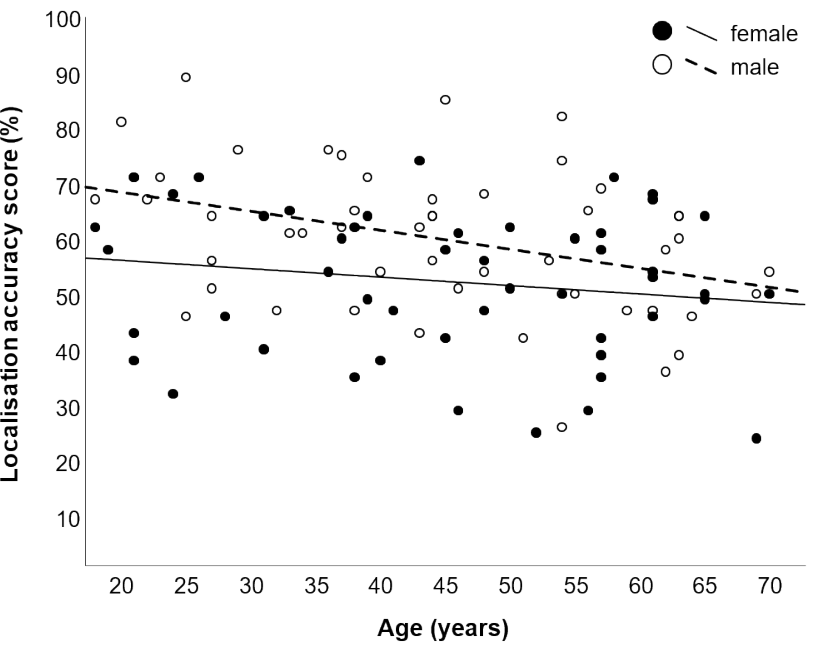

B.

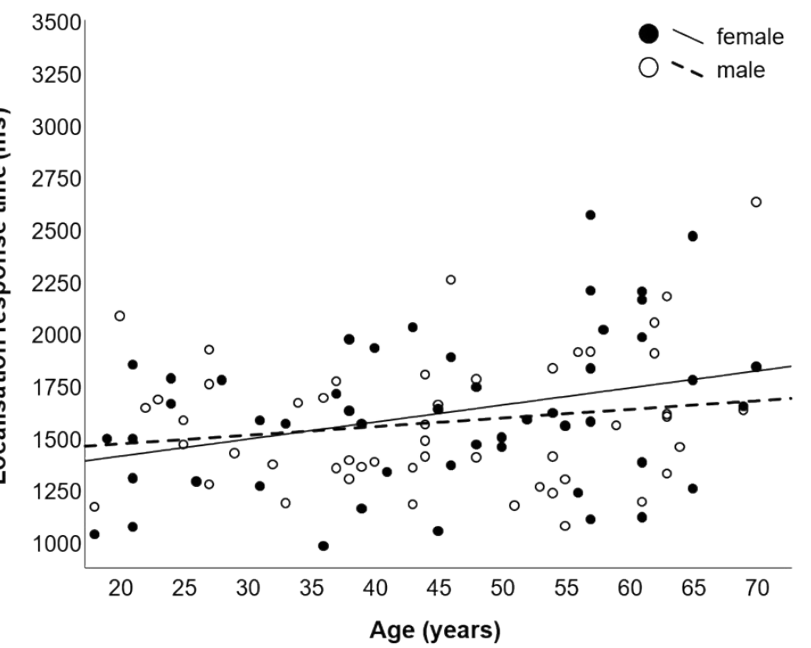

C.

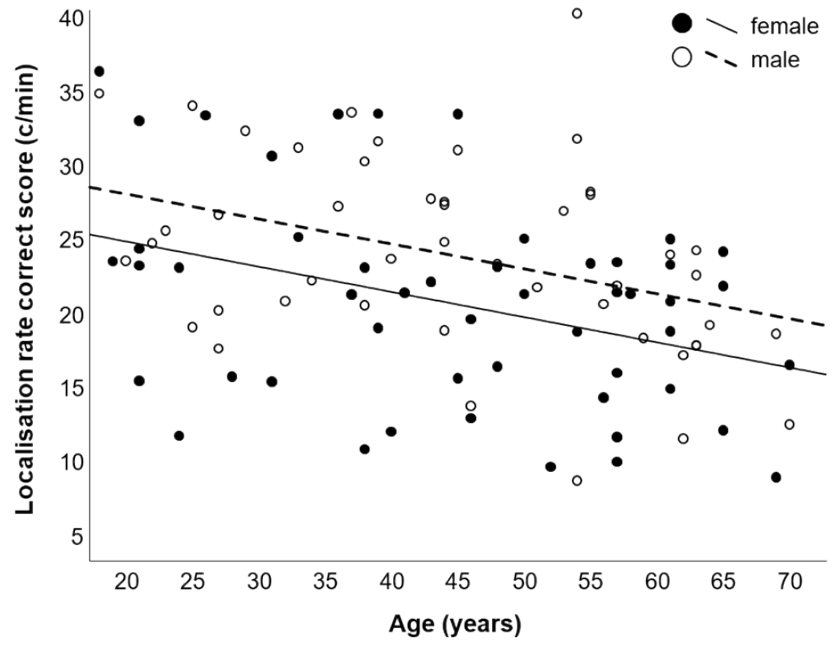

Peer) reviewing PDF | (2021:05:60922:1:0:NEW 28 Jul 2021)
Supplement 4

D.

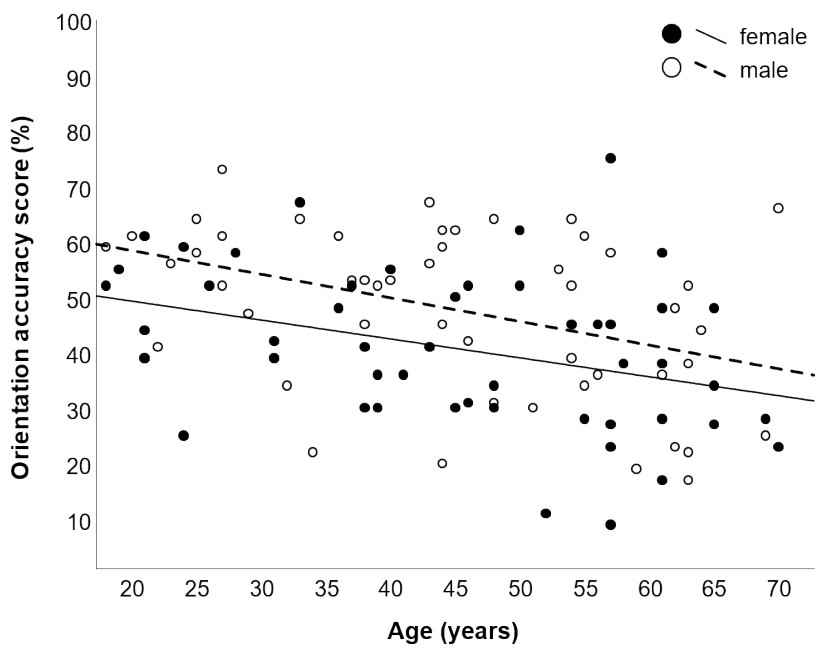

E.

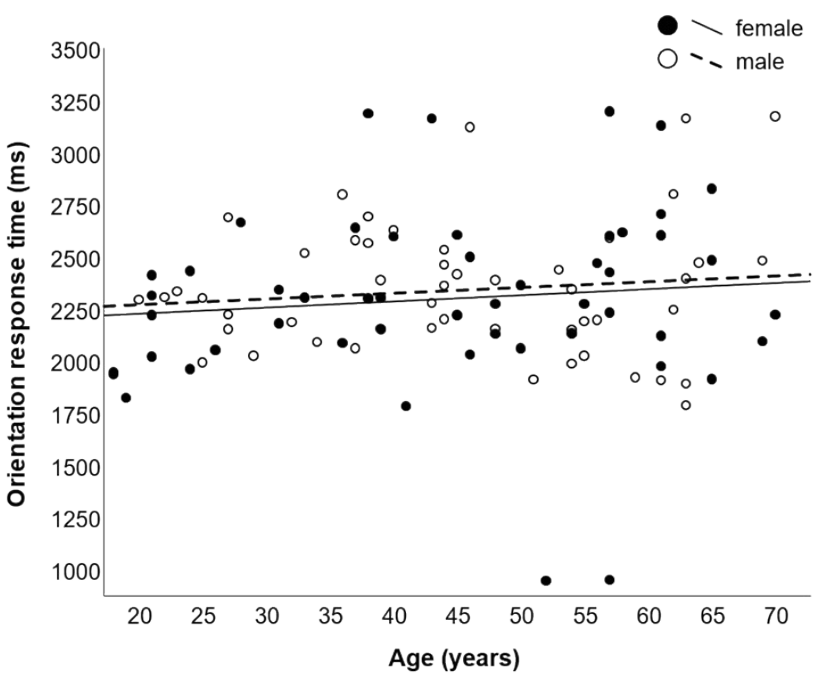

F.

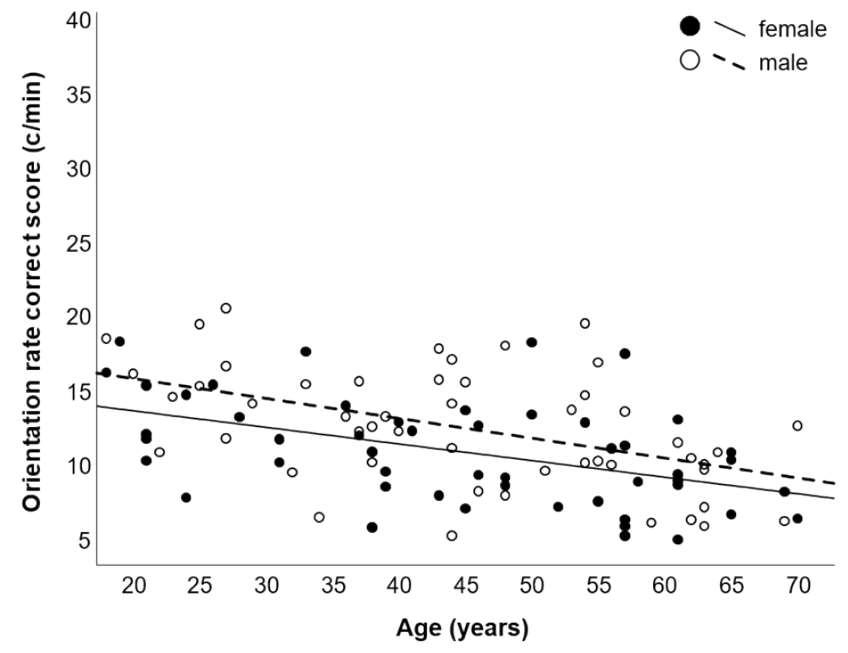


829 Supplement 4, scatter plots of iTAD localisation and orientation scores

830 Scatter plots of ITAD scores as a function of age and sex. Scores are displayed for localisation accuracy 831 (Panel A), response time (Panel B), and rate correct score (Panel C), as well as orientation accuracy 832 (Panel D), response time (Panel E), and rate correct score (Panel F). Lines represent the least squares 833 regressions. ms: milliseconds; c/min: correct responses per minute. 
834

835

A.

Supplement 5

836

837

838

839

840

841

842

843

844

845

846

847

848

849

850

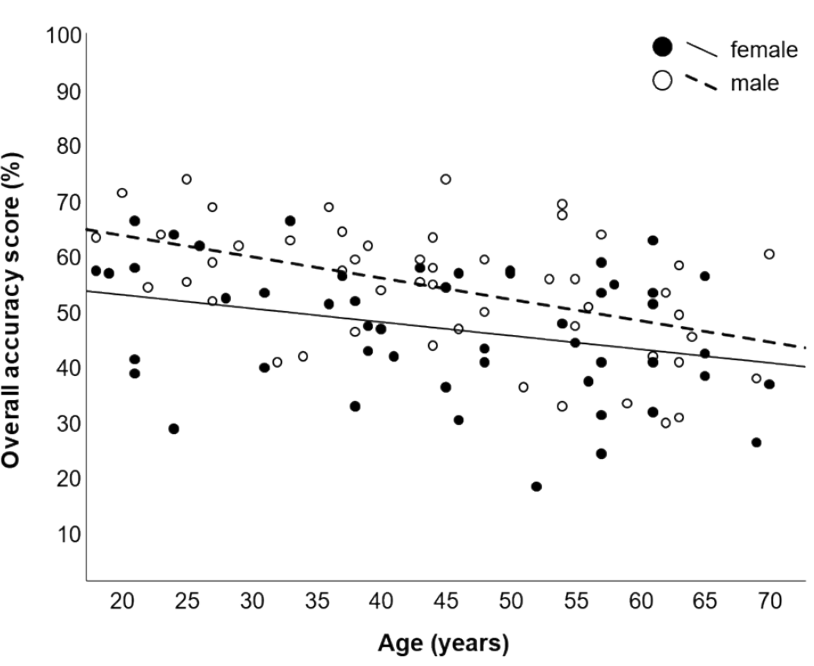

B.

851

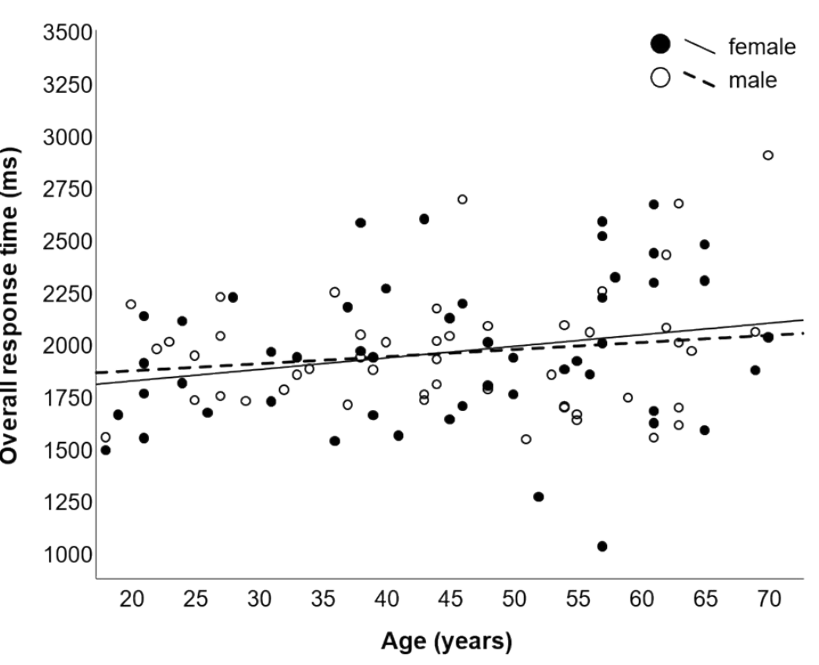

852

853

854

855

856

857

858

859

860

861

862

863

864

865

866

867

868

869

870

871

872

873

874

875

876

877

878

879

880

C.

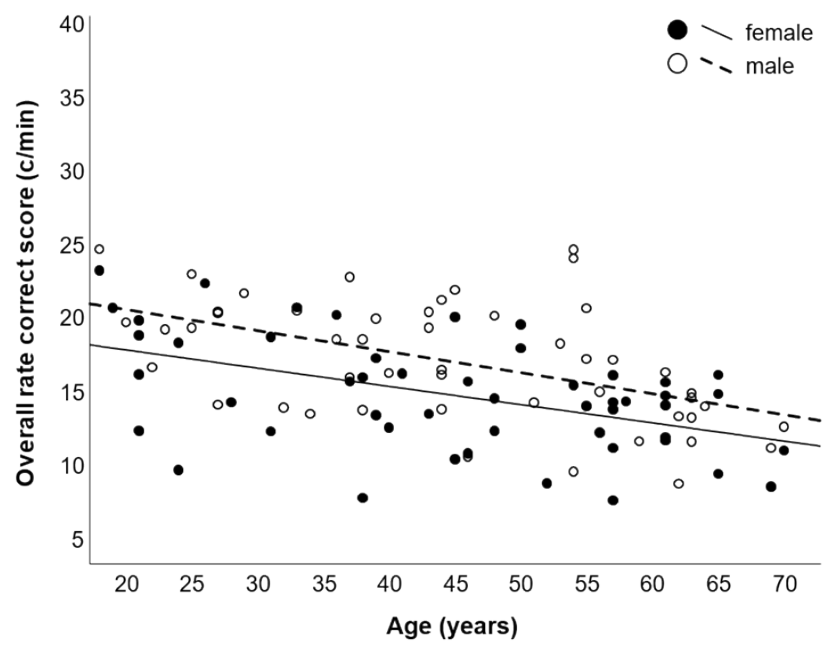

Supplement 5, scatter plots of iTAD overall scores 
881 Scatter plots of iTAD overall scores (mean of localisation and orientation scores) as a function of age and 882 sex. Scores are displayed for overall accuracy (Panel A), response time (Panel B), and rate correct score 883 (Panel C). Lines represent the least squares regressions.

884 ms: milliseconds; c/min: correct responses per minute. 
Figure 1

TPDT assessment procedure

Example of the assessment of a hypothetical two-point discrimination threshold (TPDT). Assessment is based on a forced-choice response (one or two points), alternating four runs with either increasing or decreasing caliper distances. Steps taken are either in $5 \mathrm{~mm}$. (first run) or $2 \mathrm{~mm}$. (other runs). Three consecutive reports of either one or two points indicates a reversal. Mean of the four reversals is calculated for the TPDT score.

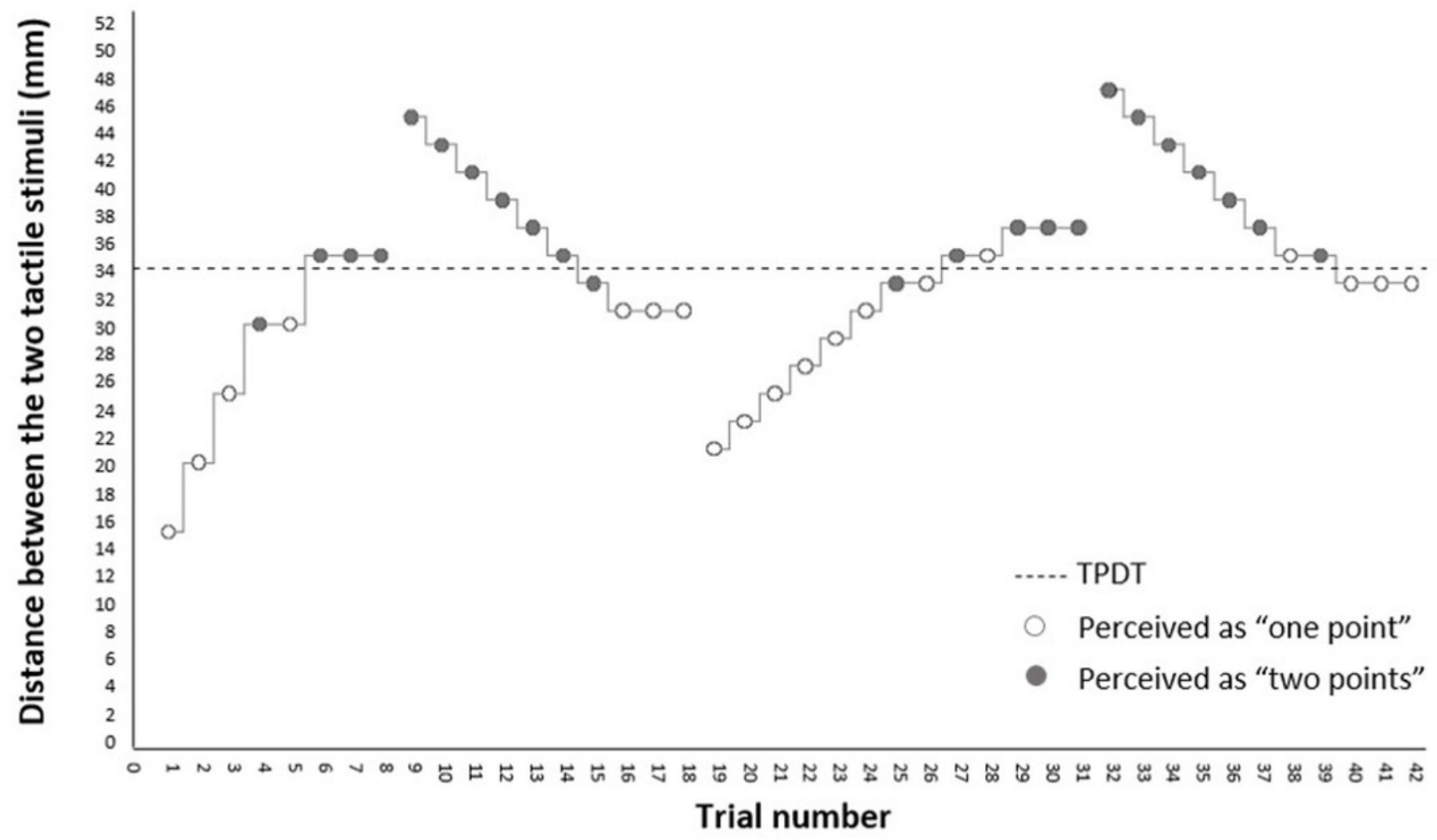




\section{Figure 2}

the imprint Tactile Acuity Device

The imprint Tactile Acuity Device (iTAD), containing twelve build-in vibrotactile stimulators (top), and wirelessly connected tablet. The iTAD performs two tactile acuity tests: 1) the localisation test (bottom left) where the perceived location of the tactile stimulus is selected and 2) the orientation test (bottom right) where the perceived location of a second tactile stimulus, relative to a first, is selected. For both tests, as well as the overall score (mean of both tests), the accuracy score (i.e. percentage correct), the average response time and the rate correct score (number of correct responses per minute of response activity) is calculated.
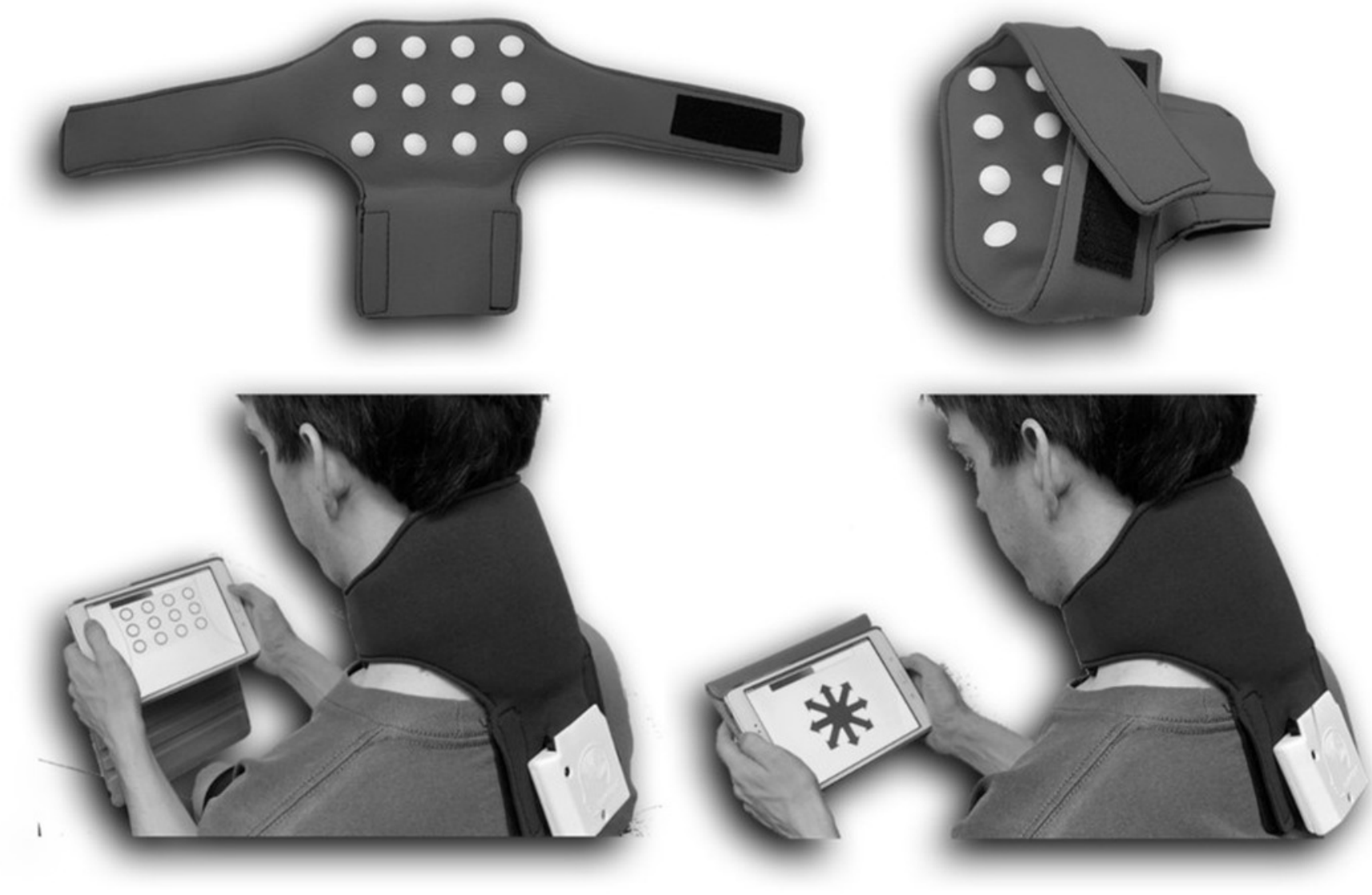


\section{Figure 3}

results sequential mediation analyses

Relationships between demographics (sex and age), anthropometrics (body mass index (BMI) and neck surface area (NSA)) and TTAD accuracy scores for the localisation test (Panel A) and orientation test (Panel B). Relationships are expressed in semi-partial correlations (sr) and unstandardized regression coefficients (b), including their level of significance (p). Coding for sex: female $=0$ and male $=1$. 
A.

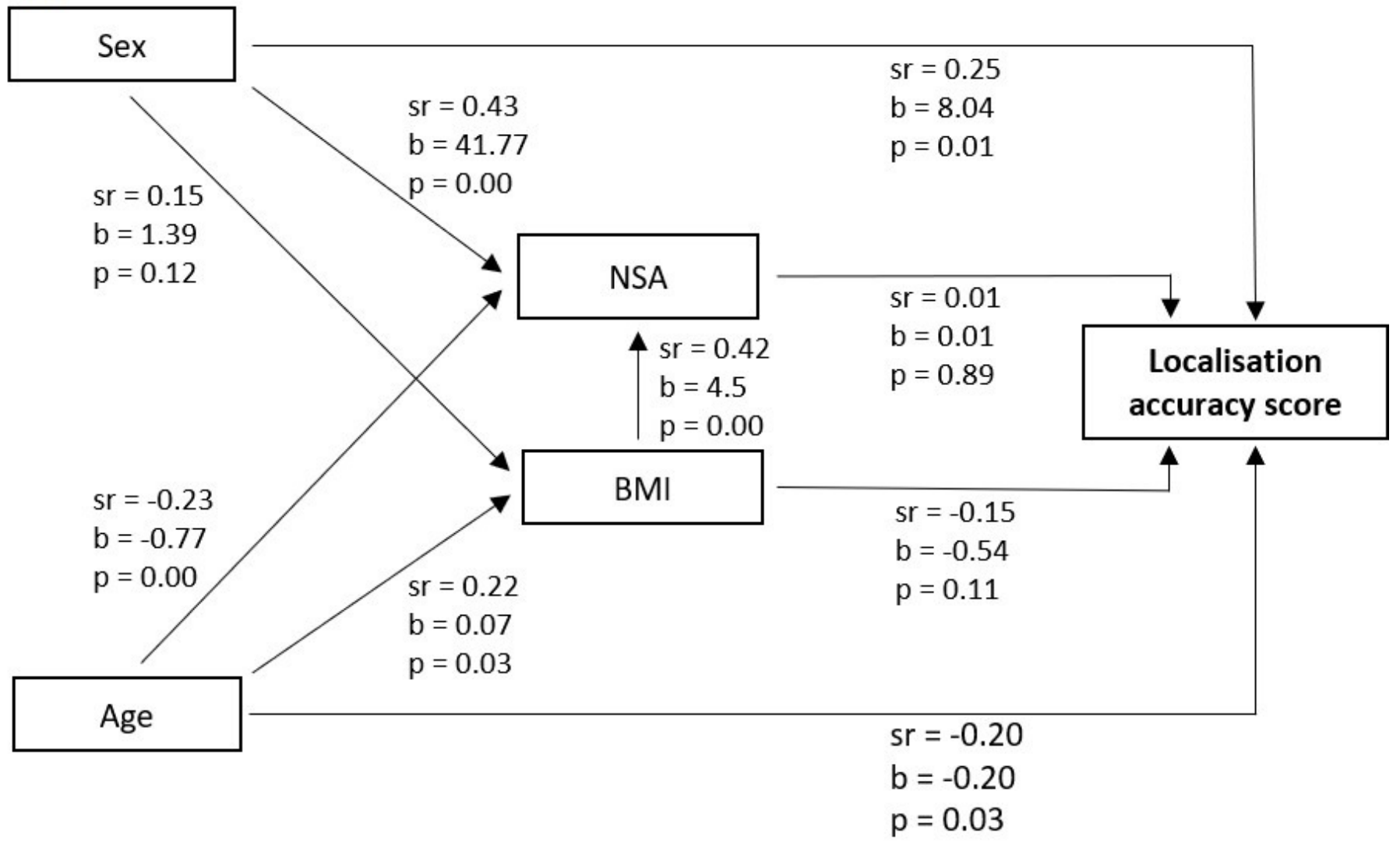

B.

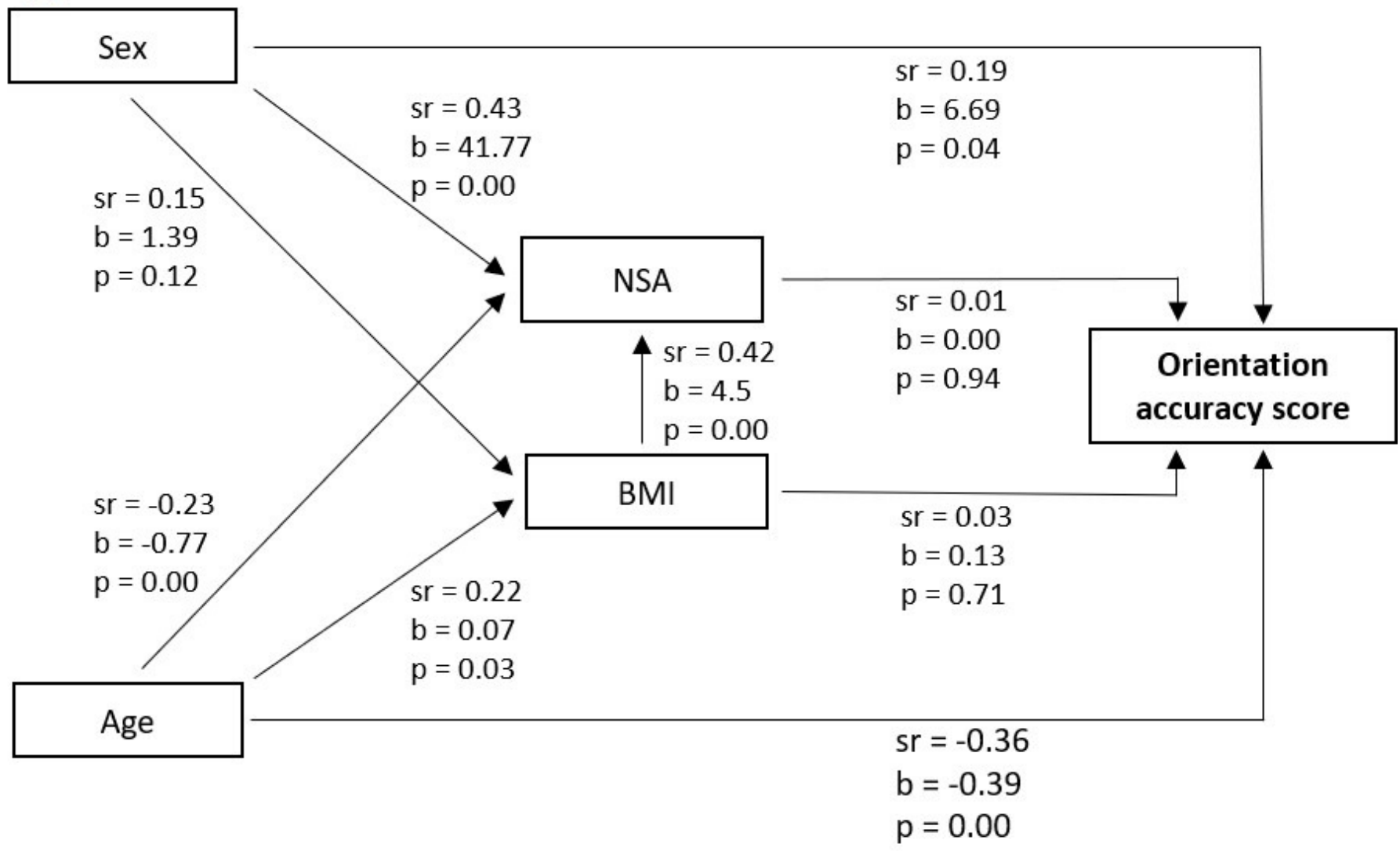


Figure 4

scatter plots of localisation and orientation accuracy scores

Scatter plots of iTAD accuracy scores as a function of age and sex. Scores are displayed for the localisation (Panel A) and orientation (Panel B) test. Lines represent the least squares regressions. 

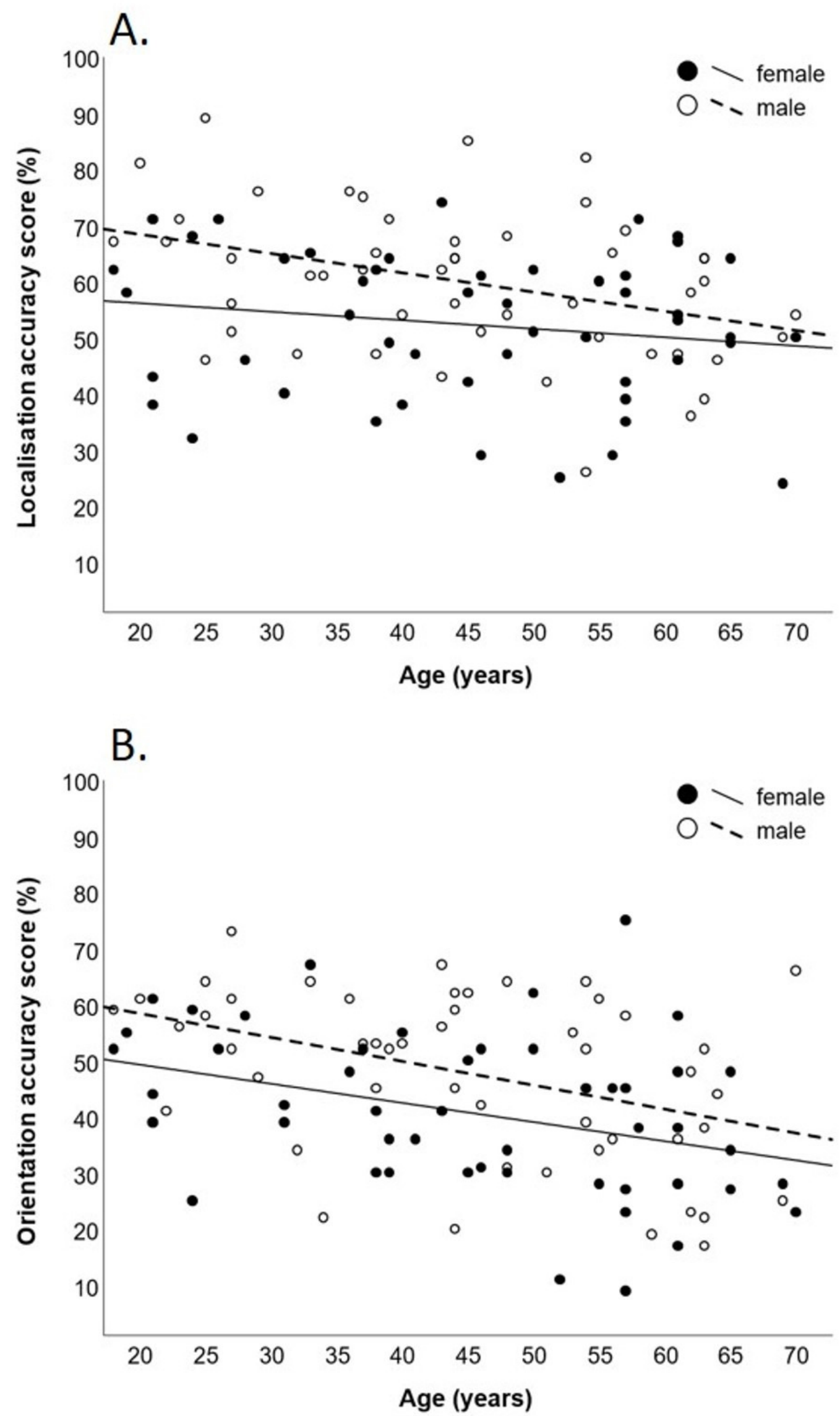


\section{Table $\mathbf{1}$ (on next page)}

Mean, inter-rater reliability and measurement error for the iTAD and two-point discrimination threshold scores.

iTAD: imprint Tactile Acuity Device; TPDT: two-point discrimination threshold; AS: accuracy score; RT: average response time; RCS: rate correct score; ms: milliseconds; c/min: correct responses per minute; ICC: intraclass correlation coefficient; SEM: standard error of measurement; CoV: coefficient of variation 
1 Table 1

2 Mean, inter-rater reliability and measurement error for the iTAD and two-point discrimination threshold 3 scores.

4

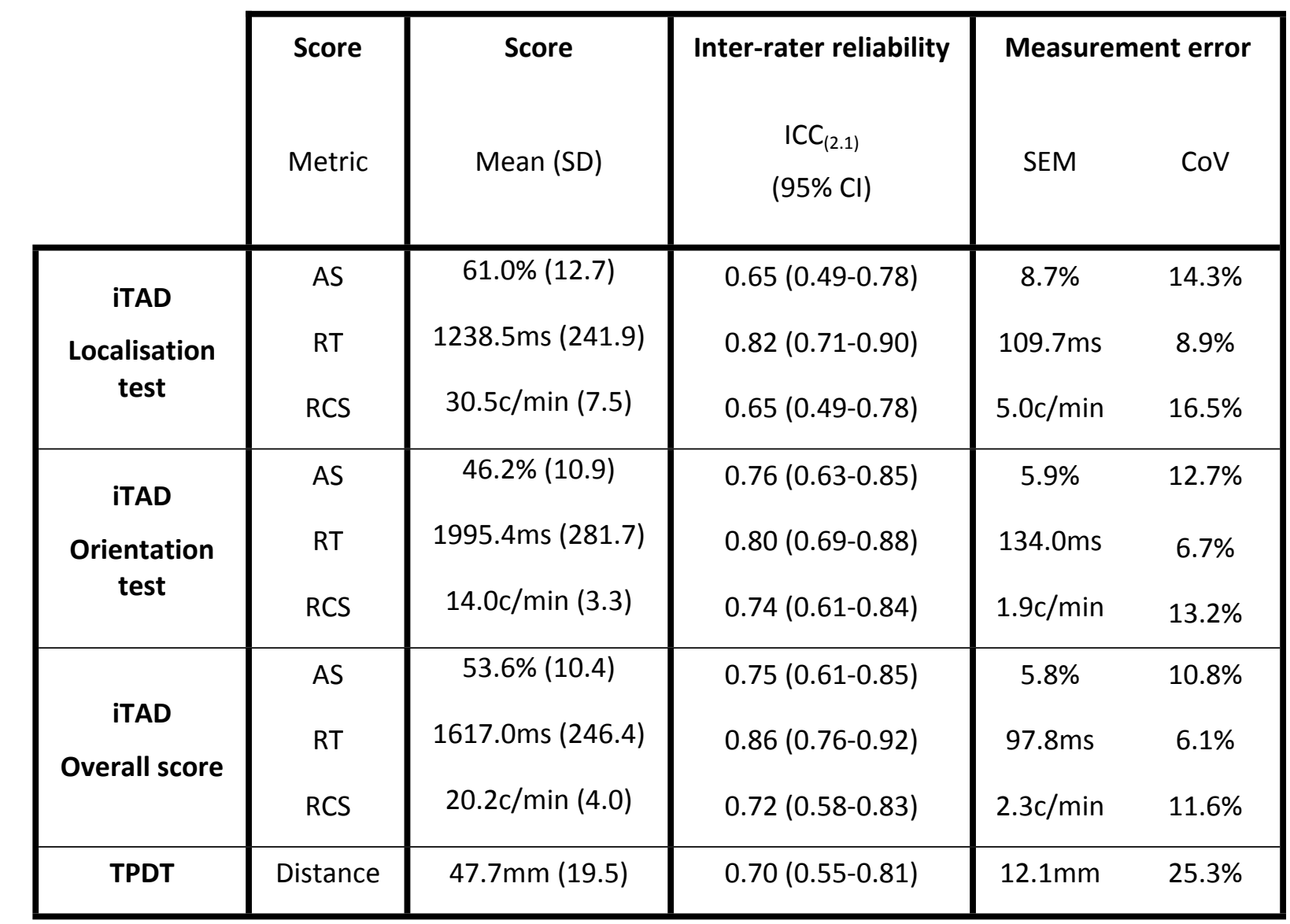

5

6 iTAD: imprint Tactile Acuity Device; TPDT: two-point discrimination threshold; AS: accuracy score; RT:

7 average response time; RCS: rate correct score; ms: milliseconds; c/min: correct responses per minute;

8 ICC: intraclass correlation coefficient; SEM: standard error of measurement; CoV: coefficient of variation 


\section{Table 2 (on next page)}

Smallest detectable changes with $80 \%, 85 \%, 90 \%$ and $95 \%$ confidence intervals for the ITAD and two-point discrimination threshold scores.

ITAD: imprint Tactile Acuity Device; TPDT: two-point discrimination threshold; AS: accuracy score; RT: average response time; RCS: rate correct score; ms: milliseconds; c/min: correct responses per minute; SDC: smallest detectable change 
1 Table 2

2 Smallest detectable changes with $80 \%, 85 \%, 90 \%$ and $95 \%$ confidence intervals for the iTAD and two-

3 point discrimination threshold scores.

4

5

6

7

8

10

11

12

13

14

15

16

17

18

19

20

21

22

23

24

25

ITAD: imprint Tactile Acuity Device; TPDT: two-point discrimination threshold; AS: accuracy score; RT:

26 average response time; RCS: rate correct score; ms: milliseconds; c/min: correct responses per minute;

27 SDC: smallest detectable change 